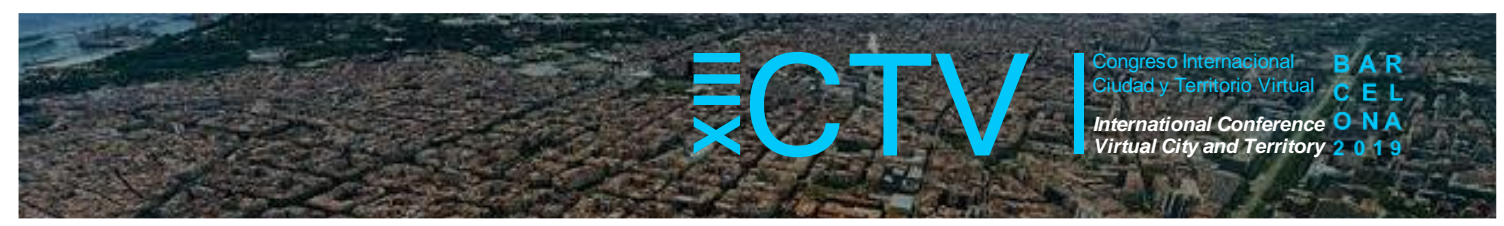

\title{
DEFINICIÓN ESPACIAL DEL EFECTO DE ENFRIAMIENTO DE LOS ESPACIOS VERDES URBANOS MEDIANTE TELEDETECCIÓN: ESTUDIOS DE CASO EN EL ÁREA METROPOLITANA DE BARCELONA
}

\author{
Arellano, Blanca ${ }^{1}$; García-Haro, Alan ${ }^{2 *}$; y Roca, Josep ${ }^{3}$ \\ Remisión inicial: 2019-09-25; Remisión definitiva: 2019-10-21; Publicación: 2019-21-21
}

Citación: Arellano, B. et al. (2019). Definición espacial del efecto de enfriamiento de los espacios verdes urbanos mediante teledetección: Estudios de caso en el Área Metropolitana de Barcelona. En XIII CTV 2019 Proceedings: XIII International Conference on Virtual City and Territory: "Challenges and paradigms of the contemporary city": UPC, Barcelona, October 2-4, 2019. Barcelona: CPSV, 2019, p. 8956. E-ISSN 2604-6512. DOI http://dx.doi.org/10.5821/ctv.8956

\section{Resumen}

Los espacios verdes urbanos juegan un papel fundamental en la adaptación de las ciudades al cambio climático. Comúnmente, la alta concentración de vegetación dentro de las ciudades, viene acompañada de un incremento de la humedad en el aire y una mayor proyección de sombras sobre las superficies. Lo que rompe la continuidad de la cubierta de suelo artificializado que caracteriza a las ciudades y la alta cantidad de radiación solar absorbida por esta, que ocasiona, en parte, la isla de calor urbana (UHI por sus siglas en inglés).

En este sentido, los espacios verdes registran una reducción de temperatura en relación a su contexto urbano y que comúnmente se extiende sobre los alrededores más cercanos. Dicho efecto es conocido como isla de frío de los espacios verdes ( $\mathrm{GCl}$ por sus siglas en inglés) y se aborda comúnmente por medio de dos indicadores de magnitud: la extensión e intensidad de enfriamiento. Por una parte, la extensión de enfriamiento ( $L$ max) se refiere a un indicador espacial que describe la distancia entre el perímetro del espacio verde y el punto más alejado de la propagación de su efecto microclimático sobre sus alrededores. Mientras que la intensidad de enfriamiento $(\Delta T)$, describe el comportamiento térmico de los espacios, al puntualizar la diferencia de temperatura entre el contexto urbano y el espacio verde. Ante esto, la literatura ha abordado la cuantificación del efecto de enfriamiento mediante tres tipos de aproximaciones al análisis del comportamiento climático de los espacios urbanos: 1) mediciones de campo, 2) modelado numérico y 3) teledetección. En general, existe un amplio consenso en la cuantificación del efecto de enfriamiento de los espacios verdes mediante el cálculo de la $\Delta T$ en los tres tipos de aproximaciones. No obstante, la definición espacial del efecto de enfriamiento ha venido evolucionando en las últimas décadas y presenta un panorama abierto a propuestas metodológicas que sean pertinentes a diferentes contextos. Particularmente, la recuperación de la Temperatura de la Superficie Terrestre (LST por sus siglas en inglés) de imágenes satelitales, ha permitido la inclusión de estudios microclimáticos de mayor escala a la discusión sobre la definición espacial de la influencia térmica de los espacios urbanos mediante aproximaciones estadísticas.

Ante esto, el presente trabajo aborda una aproximación en múltiples etapas al análisis espacial para la cuantificación del efecto de enfriamiento de los espacios verdes en el área metropolitana de Barcelona a partir de la LST del satélite Landsat-8 OLIITIRS. Se selecciona el periodo estío como caso de estudio, debido al incremento de vulnerabilidad que implica el cambio climático en las ciudades durante episodios extremos de ola de calor, que se ven acentuados por la $U H I$. Se cuantifica la $L$ max e $\Delta T$ del efecto de enfriamiento de siete espacios verdes en la conurbación de Viladecans, Gavà y Castelldefels, por medio de tres métodos analíticos basados en múltiples etapas de subdivisión espacial de los alrededores urbanos mediante anillos concéntricos. Los primeros resultados, muestran una $\Delta T$ de $1,25^{\circ} \mathrm{C}$ y $1,50^{\circ} \mathrm{C}$ en relación a los anillos concéntricos de 0-100m y 100-300m respectivamente. La Lmax calculada con anillos concéntricos de $10 \mathrm{~m}$ de ancho registraron una media de $91,67 \mathrm{~m}$ con una $\Delta T$ máxima $(\Delta T \max )$ de $1,22^{\circ} \mathrm{C}$. Por último, con secciones transversales de $10 \mathrm{~m}$ de ancho en complemento a los anillos concéntricos de una vía arborizada, se identificó una $\triangle T$ Tmax media de $2,21^{\circ} \mathrm{C}$ en zonas industriales, $1,05^{\circ} \mathrm{C}$ en áreas residenciales y $1,76^{\circ} \mathrm{C}$ en espacios adyacentes a otro parque; así como una $L$ max media de $109,00 \mathrm{~m}$ al noreste y $129,67 \mathrm{~m}$ al suroeste, con una máxima de $170,00 \mathrm{~m}$ en las zonas industriales y $310,00 \mathrm{~m}$ en el área adyacente al otro parque. La $\Delta T$ max registra una correlación de $0.81 R^{2}(p<0.01)$ con la $L S T$ media de los alrededores más cercanos al perímetro del parque, mientras que resulta en una correlación no

\footnotetext{
1 Universidad Politécnica de Cataluña, Departamento de Tecnología de la Arquitectura, Centro de Política de Suelo y Valoraciones http://orcid.org/0000-0001-7128-3667; ${ }^{2}$ https://orcid.org/0000-0002-4302-6492; ${ }^{3}$ https://orcid.org/00000003-3970-65053. * Correo de contacto: alan.garcia.haro@upc.edu
} 


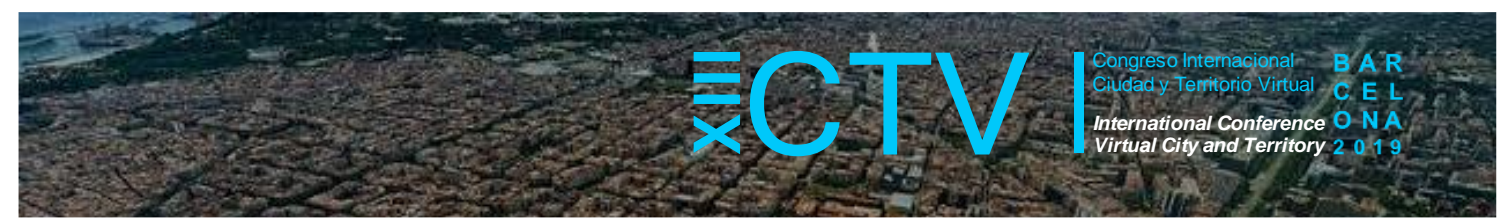

significativa con la $L S T$ de los parques. En las conclusiones se discuten las diferencias entre los métodos aplicados y las consideraciones para futuras reproducciones de los métodos en estudios de mayor escala. El presente estudio se desarrolla en el marco del proyecto "Urban-CLIMPLAN. La isla de calor urbana: efectos en el cambio climático y modelado para estrategias de planeamiento territorial y urbano. Aplicación a la Región Metropolitana de Barcelona"; financiado por el Ministerio de Economía y Competitividad de España (MINECO) y el Fondo Europeo para el Desarrollo Regional (FEDER).

\section{Abstract}

Urban green spaces play a fundamental role in the climate change adaptation of the cities. Commonly, the high concentration of vegetation within cities is accompanied by an increase in humidity in the air and a greater projection of shadows on the surfaces. Which breaks the continuity of the artificialized ground cover distinctive of the cities and the high amount of solar radiation absorbed by it, which causes, in part, the urban heat island effect (UHI).

In this sense, the green spaces register a reduction in temperature in relation to their urban context, which commonly extends over the closest surroundings. This effect is known as the urban green spaces cool island (GCl) and is commonly addressed through two indicators of magnitude: the extent and intensity of cooling. The cooling extent ( $L$ max) refers to a spatial indicator that describes the distance between the perimeter of the green space and the furthest point of the spread of its microclimatic effect on its surroundings. While the cooling intensity $(\Delta T)$ describes the temperature difference between the urban context and the green space. Given this, the literature has addressed the cooling quantification through three types of analysis of the climatic behavior of urban spaces: 1) field measurements, 2) numerical modeling and 3) remote sensing. In general, there is a broad consensus on the quantification of the cooling effect of green spaces by calculating the $\Delta T$ in the three types of approximations. However, the spatial definition of the cooling extent has been evolving in recent decades and presents an open panorama for methodological proposals that are appropriate to different contexts. Particularly, the recovery of the Land Surface Temperature (LST) from satellite images has allowed the inclusion of larger-scale microclimatic studies to discuss the spatial definition of the thermal influence of urban spaces through statistical approaches.

Given this, the present work proposes a multiple-stage approach to spatial analysis for quantifying the cooling effect of green spaces in the metropolitan area of Barcelona from the LST of the Landsat-8 OLI/TIRs satellite. We select the summer period as a case study because of the increased vulnerability posed by climate change in cities during extremes heat waves episodes, which is accentuated by the UHI. We quantify the $L$ max and $\Delta T$ of the cooling effect of seven green spaces in the conurbation of Viladecans, Gavà and Castelldefels through three analytical methods based on multiple stages of spatial subdivision of urban surroundings by concentric rings. The first results show a $\Delta T$ of $1.25^{\circ} \mathrm{C}$ and $1.50^{\circ} \mathrm{C}$ in relation to the concentric rings of $0-100 \mathrm{~m}$ and $100-300 \mathrm{~m}$ respectively. The Lmax calculated with the $10 \mathrm{~m}$-width concentric rings registered an average of $91.67 \mathrm{~m}$ with a maximum $\Delta T(\Delta T \max )$ of $1.22^{\circ} \mathrm{C}$. Finally, with $10 \mathrm{~m}$-width cross sections in addition to the concentric rings of an arborized street, we identify an average $\triangle T$ max of $2.21^{\circ} \mathrm{C}$ in industrial areas, $1.05^{\circ} \mathrm{C}$ in residential areas and $1.76^{\circ} \mathrm{C}$ in spaces adjacent to another park. As well as an average $L \max$ of $109.00 \mathrm{~m}$ to the northeast and $129.67 \mathrm{~m}$ to the southwest, with a maximum of $170.00 \mathrm{~m}$ in the industrial areas and $310.00 \mathrm{~m}$ in the area adjacent to the other park. The $\Delta$ Tmax records a correlation of $0.81 \mathrm{R}^{2}(p<0.01)$ with the average LST of the closest surroundings to the perimeter of the park, while resulting in a non-significant correlation with the LST of the parks. In the conclusions, we discuss the differences between the methods applied and the considerations for their reproduction in larger-scale studies. The present study is part of the project "Urban-CLIMPLAN. The urban heat island: effects on climate change and modeling for territorial and urban planning strategies. Application to the Metropolitan Region of Barcelona"; financed by the Ministry of Economy and Competitiveness of Spain (MINECO) and the European Fund for Regional Development (FEDR).

Palabras Clave: isla de frío de los parques urbanos; isla de calor urbana: microclima urbano

Key words: urban parks cool island; urban heat island; urban microclimate

\section{Introducción}

Los parques urbanos juegan un papel fundamental en la adaptación al cambio climático de las ciudades. Comúnmente con la mayor concentración de vegetación y superficies permeables en las ciudades, los parques derivan en una mayor humedad en el aire y sombras proyectadas sobre las superficies. Dichas características rompen la continuidad de la Isla de Calor Urbana (UHI por sus siglas en inglés), que es el incremento de la temperatura en las ciudades causado, en parte, 


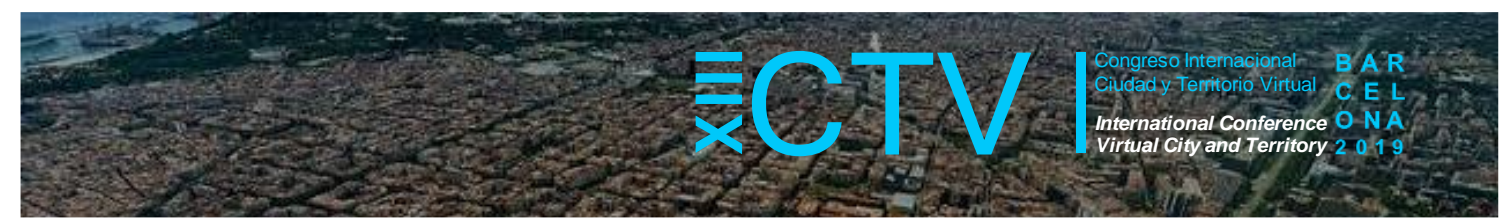

por la alta absorción del calor directo de la radiación solar por las superficies artificiales (Oke, 1982). Por lo tanto, los parques registran temperaturas más bajas que el resto de los espacios urbanos y generan un efecto de enfriamiento que se extiende a su entorno creando un efecto de "isla de frío" (Spronken-Smith y Oke, 1998). En este sentido, los estudios apuntan a cuantificar la influencia de las características físicas de los parques y sus alrededores urbanos, buscando mejorar los criterios para un diseño y planificación urbanos sensibles al clima (Norton, Coutts, Livesley, Harris, Hunter y Williams, 2015; Bowler, Buyung-Ali, Knight y Pullin, 2010). Donde al paliar la UHI (Feyisa, Dons y Meilby, 2014), también se reducen los riesgos a la salud de los habitantes durante los períodos extremos de calor en las ciudades (Arbuthnott y Hajat, 2017; Patz, Campbell-Lendrum, Holloway y Foley, 2005).

El efecto de enfriamiento de los parques se cuantifica por el límite de la extensión de enfriamiento, que es la distancia máxima alcanzada por la caída de temperatura fuera de los límites del parque; y la intensidad de enfriamiento, que es la diferencia de temperatura entre el parque y el espacio urbano en sus alrededores (Jauregui, 1990; Spronken-Smith y Oke, 1998). Las divergencias en el cálculo de estos últimos indicadores en la literatura están relacionadas con la escala del estudio, la distribución espacial de los datos y el método aplicado para registrar las temperaturas. En cuanto a la intensidad de enfriamiento, existe un consenso sobre su cálculo, pero con ligeras diferencias en la atribución de la temperatura que representa el parque y la que representa el espacio urbano. La extensión de enfriamiento presenta un consenso más bajo sobre su definición, con una mayor dependencia del método aplicado y la escala de estudio. Investigaciones anteriores señalaron que la extensión de enfriamiento de los parques entre 3 y 200 ha de tamaño, está en el rango de 50 a 300 metros $(\mathrm{m})$, pero los parques más grandes van de 200 a $2000 \mathrm{~m}$. Mientras que la intensidad de enfriamiento registra valores entre 1 y $4{ }^{\circ} \mathrm{C}$ durante el día y entre 2 y $5^{\circ} \mathrm{C}$ por la noche (Kuttler, 2012), con una intensidad media entre 0.94 y $1.15^{\circ} \mathrm{C}$ (Bowler, et al., 2010).

Por otro lado, los estudios han señalado una influencia dividida entre las características físicas propias de los parques y las de sus alrededores. Incluso cuando la vegetación es la característica más influyente (Yuan, Emura y Farnham, 2017), la presencia de cuerpos de agua (Hathway y Sharples, 2012), el porcentaje de superficies sin sellar, el tamaño de los parques, su forma (Cheng, Wei, Chen, Li y Song, 2015) y la distribución de sus elementos del paisaje (Cao, et al., 2010; Yang, et al., 2017), han sido identificados como elementos que también definen el efecto de enfriamiento de los parques, ya sea como parte de los parques o sus alrededores. De modo que, algunos estudios analizan la influencia de la densidad edificada, la distancia a grandes cuerpos de agua y la distancia a áreas naturales sobre el efecto de enfriamiento (Jauregui, 1990; Hamada, Tanaka y Ohta, 2013; Cheng, et al., 2015)-

Las investigaciones anteriores dividen los estudios en dos etapas metodológicas básicas: 1) adquisición de datos de temperatura y 2) evaluación del efecto de enfriamiento. En particular, los métodos para la adquisición de datos de temperatura pueden concentrarse en cuatro grupos. Primero, la investigación de las variaciones de temperatura del aire con las estaciones meteorológicas y su relación con la distancia a los espacios verdes, limitada al análisis a gran escala y con necesidad de interpolar la información (Jauregui, 1990; Anjos y Lopes, 2017). Otros estudios realizan campañas de medición de campo con estaciones meteorológicas portátiles en rutas que cruzan los parques y sus alrededores, lo que se limita a casos específicos (SpronkenSmith y Oke, 1998; Yan, Wu y Dong, 2018). Las últimas décadas han aumentado los estudios que recurren al modelado numérico y la simulación térmica de parques y sus alrededores en 


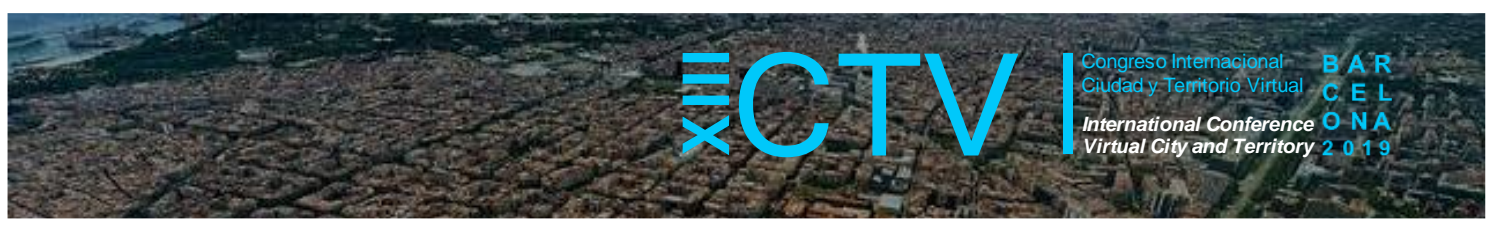

escenarios actuales y propuestos para medir la influencia microclimática de atributos físicos específicos (Chow, Pope, Martin y Brazel, 2011; Declet-Barreto, Brazel, Martin y Chow, 2013). En los últimos años, la recuperación de la Temperatura de la Superficie Terrestre (LST) mediante imágenes de teledetección se ha convertido en uno de los métodos más frecuentes aplicados al análisis multiescala del UHI o en estudios generales de climatología urbana, debido al potencial de la continuidad espacial de los datos térmicos simultáneos (Ren, et al., 2013; Cao, Onishi, Chen e Imura, 2010).

\section{1 Área de estudio}

En particular, este estudio presenta la adaptación de métodos analíticos de teledetección para la cuantificación del efecto de enfriamiento de siete parques dentro de la conurbación de Viladecans, Gavà y Castelldefels (Área VGC) (Figura 1). Un área de tres municipios y 6,431.23 hectáreas (ha), ubicada a diez kilómetros al oeste de la ciudad de Barcelona en la costa con Mediterráneo (norte a $41^{\circ} 20^{\prime} 16.6 " \mathrm{~N}$; sur a $41^{\circ} 15^{\prime} 50.8 " \mathrm{~N}$; oeste a $1^{\circ} 55^{\prime} 29.8^{\prime \prime} \mathrm{E}$; y este $2^{\circ} 04^{\prime} 26.9^{\prime \prime} \mathrm{E}$ ). Los polígonos de los parques y los municipios fueron definidos por la administración (Barcelona Regional, 2018). Se acude a la clasificación de Corine Land Cover (CLC) de 2012 como visión general de las características del área de estudio (Copernicus Land Monitoring Service, 2016).

Figura 1. Ubicación de los parques seleccionados y CLC del Área VGC

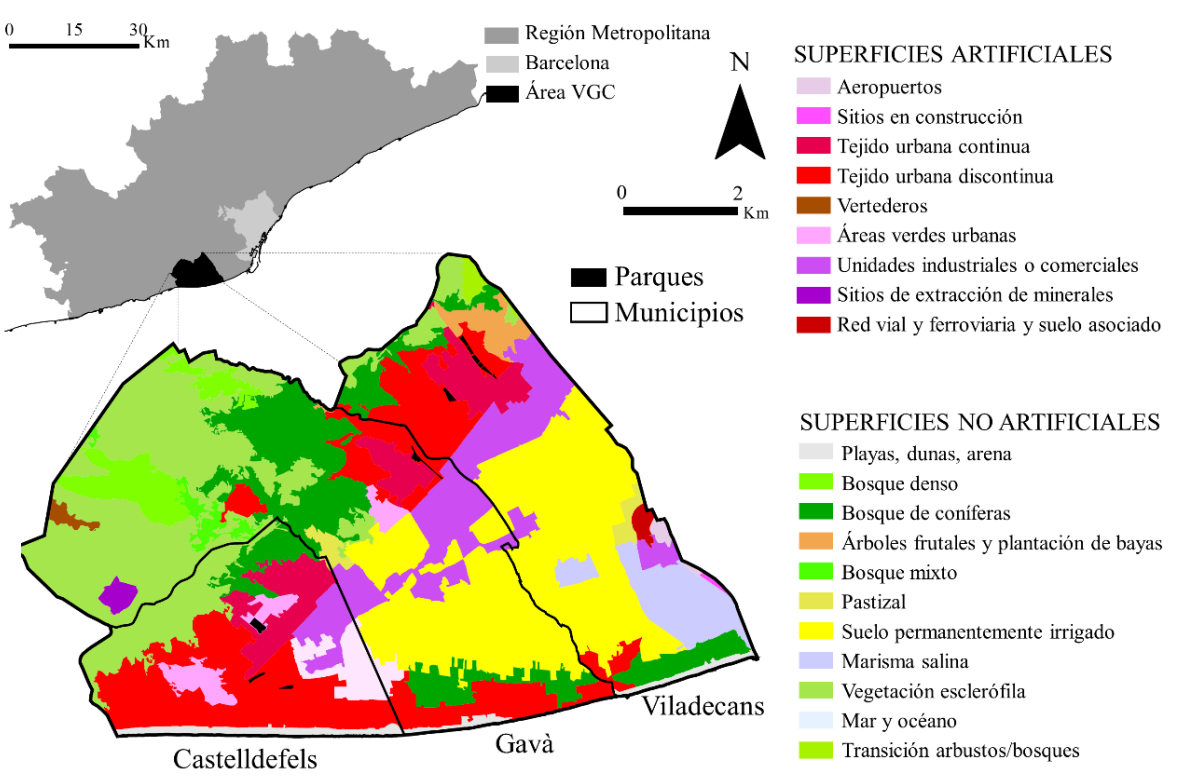

Fuente: Elaboración propia con datos de Barcelona Regional (2018) y Copernicus Land Monitoring Service, (2016)

Estudios previos han identificado una intensidad máxima de $\mathrm{UHI}$ de aire de $8^{\circ} \mathrm{C}$ en el contexto metropolitano de Barcelona (Carreras, Marín, Martín-Vide, Moreno y Sabí, 1990) y $5.8^{\circ} \mathrm{C}$ dentro de la ciudad central (Martin-Vide, Cordobilla, Moreno y Montlleó, 2015), ambos en una noche de invierno. Por otro lado, con la LST se registró una diferencia entre las áreas urbanas y rurales de $4.28^{\circ} \mathrm{C}$ durante el día y $2.56^{\circ} \mathrm{C}$ por la noche en otoño (Arellano y Roca, 2016).

Del mismo modo, los estudios sobre el efecto de enfriamiento de los parques a través de mediciones de campo en Barcelona han identificado una intensidad de $2^{\circ} \mathrm{C}$ y una extensión de $100 \mathrm{~m}$ en Parc Turó de 3 hectáreas (ha) dentro de un tejido urbano denso. Mientras que, con 30 


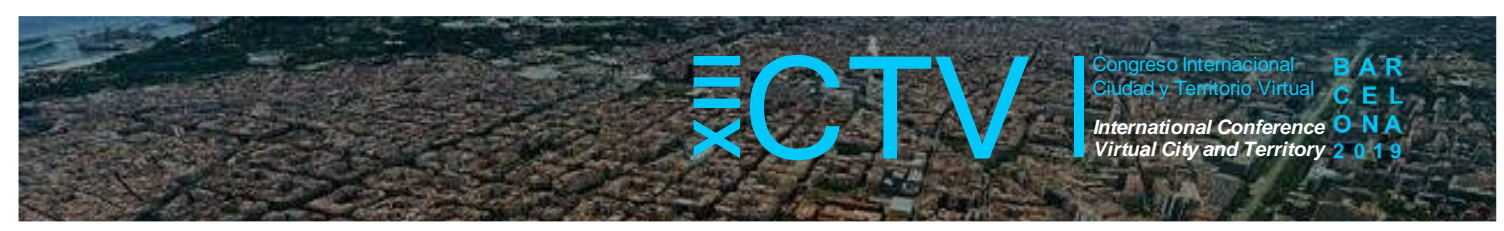

ha y un entorno urbano menos compacto, el Parc de la Ciutadella también registró una intensidad de $2^{\circ} \mathrm{C}$, pero con una extensión de enfriamiento de más de $150 \mathrm{~m}$ (Martin-Vide, et al., 2015). Además, una evaluación simultánea de la influencia del diseño de dos parques en la misma ubicación, identificó que las áreas con vegetación más densa reducen la temperatura del aire alrededor de $5^{\circ} \mathrm{C}$ durante el día y $3^{\circ} \mathrm{C}$ por la noche, así como una diferencia de temperatura de superficie de $13.2^{\circ} \mathrm{C}$ en superficies permeables durante el día y $2.21^{\circ} \mathrm{C}$ por la noche (Arellano y Roca, 2017).

Figura 2. UHI de la región metropolitana de Barcelona de LST nocturna

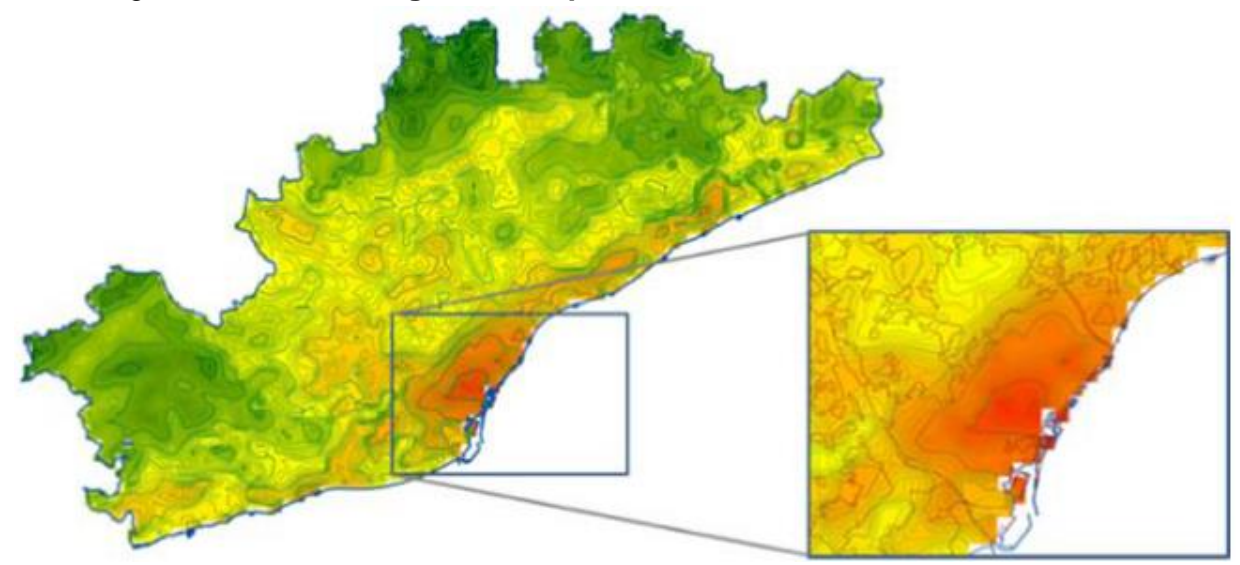

Fuente: Tomada de Arellano y Roca (2018).

El área de VGC presenta múltiples variaciones de la cubierta del suelo (Figura 1) y es parte de la UHI que se vislumbra en la costa, alrededor de la ciudad de Barcelona (Figura 2). Los tres municipios presentan tejido urbano continuo y discontinuo y unidades industriales. Castelldefels está cubierto principalmente por tejido urbano discontinuo; Gavà es predominantemente agrícola y natural con unidades industriales tan grandes como el tejido urbano; y Viladecans está cubierto principalmente por tierras agrícolas.

Los siete casos de estudio corresponden a áreas verdes que la administración ha identificado como puntos de interés debido a su valor paisajístico y diferencias en el contexto urbano de cada una de ellas (Barcelona Regional, 2018). Incluso cuando la administración no los identifica como parques, los siete casos presentan características de parques urbanos, por lo que en este documento serán referido como "parques" cuando se mencionen en conjunto.

Los casos de estudio son:

- Parc de la Riera de Sant Climent, Viladecans (PRSC). Situado sobre un arroyo, tiene forma alargada y colinda con áreas naturales al noreste y tejido urbano al suroeste.

- Parc Torrent Ballester, Viladecans (PTB). Rodeado por tejido urbano, tiene una forma rectangular que corresponde con el tejido urbano continuo y discontinuo.

- Parc Torre Lluch, Gavà (PTL). También inmerso en el tejido urbano, éste tiene la peculiaridad de colindar con la Rambla de Gavà al noreste.

- Rambla de Gavà, Gavà (RG). Es una calle peatonal estrecha y alargada con una densa copa de árboles. Debido a su larga extensión, está rodeado de tejido urbano continuo y discontinuo, unidades industriales y áreas verdes.

- Parc de la Muntanyeta, Castelldefels (PM). Con una forma rectangular, se encuentra en la intersección del tejido urbano continuo y discontinuo, y áreas verdes urbanas. 


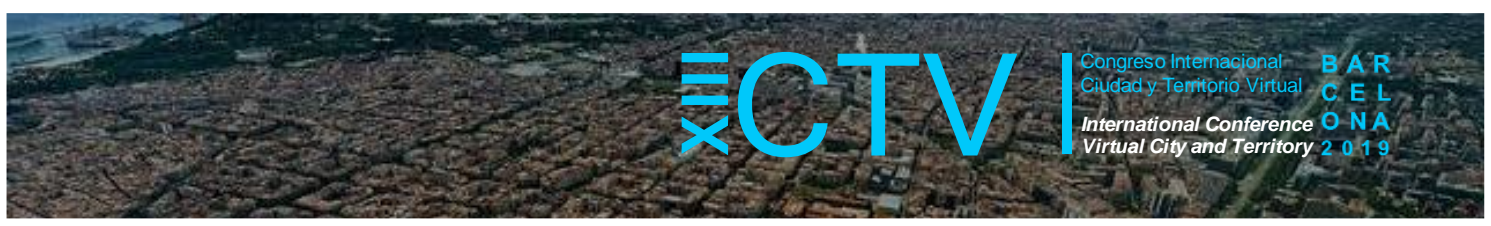

- Parc dels Tellinaires, Castelldefels (PT). De forma rectangular, está dentro de un tejido urbano discontinuo y está bordeado por un ferrocarril y una carretera hacia el norte.

- Parc de la Plaça d'Asturias, Castelldefels (PPA). Ubicado dentro de un tejido urbano discontinuo, está rodeado de edificios de viviendas de densidad media que definen su perímetro.

Figura 3. Imagen satelital de los parques seleccionados

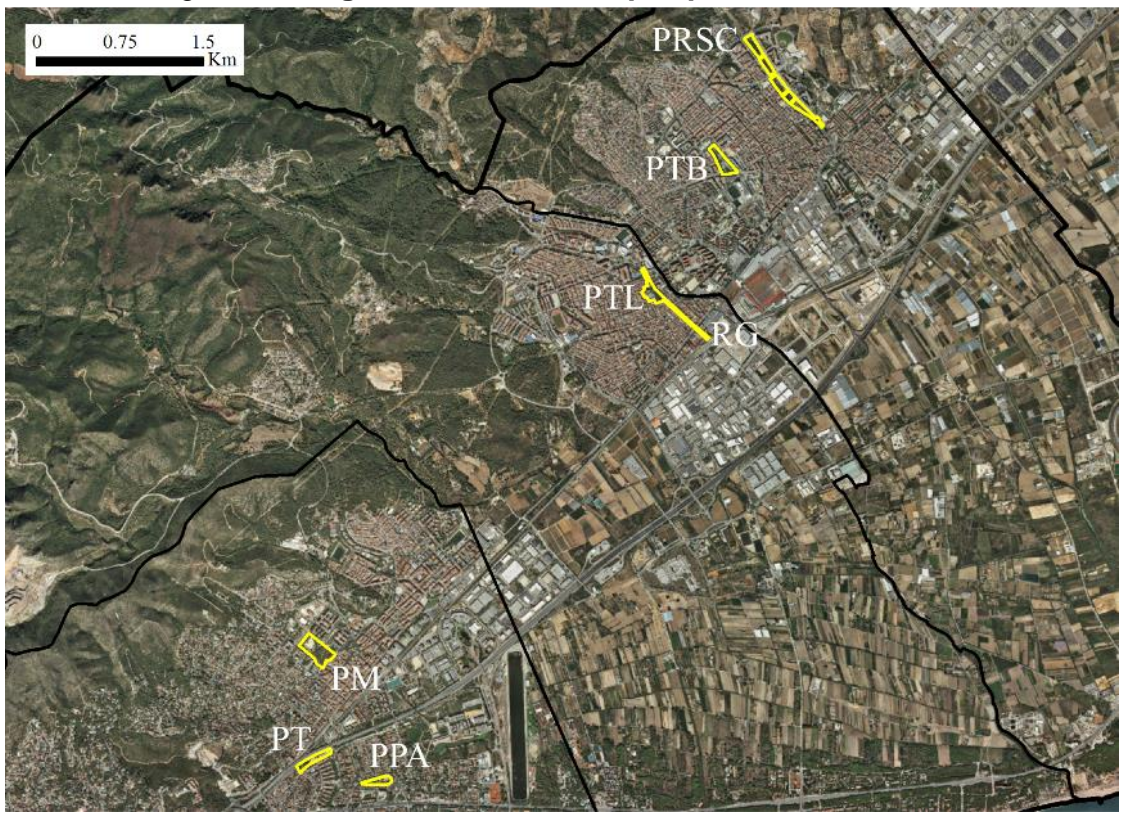

Fuente: Elaboración propia con información de Barcelona Regional (2018) y Esri (2019).

\section{Datos y métodos}

Este estudio busca cuantificar el efecto de enfriamiento de los parques a través de métodos analíticos de la distribución espacial de la LST en los parques y sus alrededores durante verano. Esta exploración de métodos tiene el propósito de definir criterios básicos para una replicación a gran escala en la región metropolitana de Barcelona. En particular, una vez que se obtiene la LST del área de estudio, se desarrollan tres métodos de diferente nivel de detalle:

- Delimitación de las áreas circundantes para un reconocimiento particular de la diferencia de temperaturas entre los parques y su contexto urbano cercano.

- Homogeneización de la temperatura del entorno a través de anillos concéntricos para cuantificar la extensión e intensidad del efecto de enfriamiento de los parques.

- Cuantificación detallada del efecto de enfriamiento del parque en diferentes orientaciones y contextos urbanos a través de secciones transversales.

La detección remota y el procesamiento de datos SIG se realiza con las herramientas ArcGIS.

\subsection{Recuperación de la LST}

La LST se recupera de las imágenes de Landsat-8 del 26 de julio de 2018 adquiridas a las 10:36GTM+1 (U.S.Geological Survey [USGS], 2018). Seleccionadas debido a la resolución de 


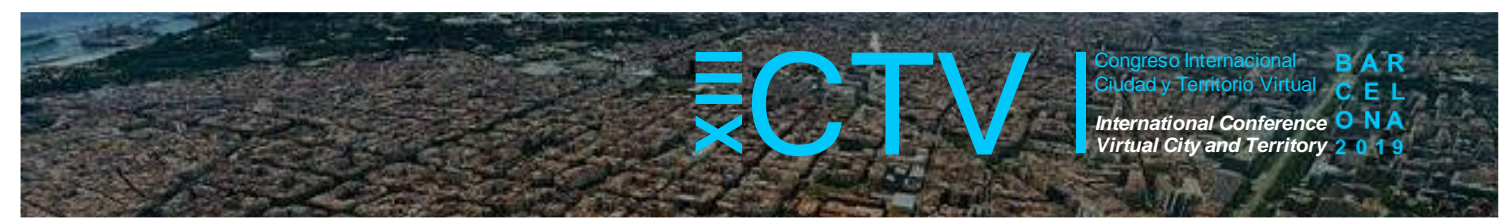

30m-pixel, adecuada al detalle propuesto del estudio y con disponibilidad de una imagen sin nubes del área de estudio de un día dentro de la semana más cálida de 2018 en Barcelona. La conversión de números digitales (DN) a radiancia de la banda de infrarroja térmica (TIR1-10) y reflectancia de las bandas roja (R-4) e infrarroja cercana (NIR-5), es realizada de acuerdo con la guía de USGS (2016). La banda TIR1 se selecciona debido a la contaminación lumínica en la banda TIR2 (Barsi, Schott, Hook, Raqueno, Markham y Radocinski, 2014), y las bandas R-4 y NIR-5 se usan para calcular el Índice de Vegetación de Diferencia Normalizada (NDVI por sus siglas en inglés), necesario para calcular la Emisividad de la superficie terrestre (LSE por sus siglas en inglés) a través del método de umbrales de NDVI (Sobrino, Jiménez-Muñoz y Paolini, 2004), requerido en la recuperación de LST. EI NDVI se calcula como [( $\rho N I R-\rho R E D) /(\rho N I R+$ $\rho R E D)]$, donde $\rho$ es la reflectancia de la banda.

La recuperación de la LST se realiza mediante el método de corrección de emisividad (Ec. 1) de Artis y Carnahan (1982):

$$
T_{B} /\left[1+\left(\lambda \times T_{B} / \alpha\right) \ln \varepsilon\right]-273.15
$$

donde $T_{B}$ es la temperatura de brillo del sensor en ${ }^{\circ} \mathrm{K}$, calculada como $\left[\mathrm{T}_{\mathrm{B}}=\mathrm{K} 2 /\right.$ / $\left.\ln (\mathrm{K} 1 / \mathrm{L} \lambda+1)\right]$, donde $\mathrm{K} 1$ y K2 son la constante de conversión térmica 1 y 2 expresada en los metadatos, y $\mathrm{L} \lambda$ es la radiancia de la banda térmica. Mientras que $\lambda$ es la longitud de onda central de la banda TIR-10 [ $(\lambda \min =10.60 ; \lambda \max =11.19)=10.895 \mu \mathrm{m}](U S G S, 2016)$ y $\alpha$ es la constante de radiación superficial $\left(\alpha=1.4388 \times 10^{-2} \mathrm{mK}\right)$ derivada de $[\alpha=(h \times c) / \sigma]$, donde $\mathrm{h}=$ constante de Planck $\left(6.62607004 \times 10^{-34} \mathrm{~J} / \mathrm{K}\right), \mathrm{c}=$ velocidad de la luz $\left(2.998 \times 10^{8} \mathrm{~m} / \mathrm{s}\right)$ y $\sigma=$ constante de Boltzmann $\left(1.38064852 \times 10^{-23} \mathrm{Js}\right)$. Finalmente, $\varepsilon$ es la LSE, en este caso calculada con el método de umbral NDVI. La LST resultante está en ${ }^{\circ} \mathrm{C}$ con una resolución de $30 \mathrm{~m}$ píxeles.

El cálculo de LSE $(\varepsilon)$ por el método de umbrales de NDVI parte de la integración de la emisividad del suelo desnudo, las superficies con vegetación y las áreas mixtas (Sobrino, Jiménez-Muñoz y Paolini, 2004). Se expresa como $\varepsilon=\varepsilon s \lambda, \varepsilon \mathrm{M} \lambda, \varepsilon \vee \lambda$, donde $\varepsilon s \lambda$ es la emisividad del suelo desnudo de las superficies con $N D V l<0.2$ y $\varepsilon \vee \lambda$ es la emisividad de las superficies con vegetación con $\mathrm{NDVI}>0.5$. Mientras que $\varepsilon_{\mathrm{M} \lambda}$ es la emisividad de superficies con cubierta mixta calculada como $[(m \times \mathrm{Pv})+n]$ para superficies con NDVI<0.5 y NDVl>0.2.

El Pv es el valor fraccional de vegetación, $\mathrm{PV}=\left[\left(\mathrm{NDVI}-\mathrm{NDVI} I_{\min }\right) /\left(\mathrm{NDV} I_{\max }-N D V I_{\min }\right)\right]^{2} ;$ donde $N D V I_{\text {min }}$ es el umbral inferior con el suelo desnudo (0.2) y NDVI max es el umbral superior con las superficies totalmente cubiertas de vegetación (0.5). Los valores de $m$ y $n$ corresponden a la relación del factor de forma de las superficies con vegetación y suelo desnudo en las áreas mixtas. Para este estudio, los valores de emisividad y los factores de forma se toman de Stathopoulou y Cartalis (2007), calculados en un contexto similar al área de estudio con el método de Sobrino et al. (2004), donde se obtuvo que $\varepsilon_{\mathrm{s} \lambda}=0.95 ; \varepsilon v_{\lambda}=0.98 ; m=0.017$; y $n=$ 0.963 .

\subsection{Indicadores del efecto de enfriamiento}

El efecto de enfriamiento se calcula por su extensión e intensidad. La intensidad de enfriamiento $(\Delta T)$ es la diferencia entre la temperatura de los parques $\left(T_{P}\right)$ y las áreas urbanas ( $T u$ ), calculada como Tu - TP (Spronken-Smith y Oke, 1998) y medida en grados Celsius $\left({ }^{\circ} \mathrm{C}\right)$. El efecto de enfriamiento positivo muestra valores positivos de $\Delta T$. 


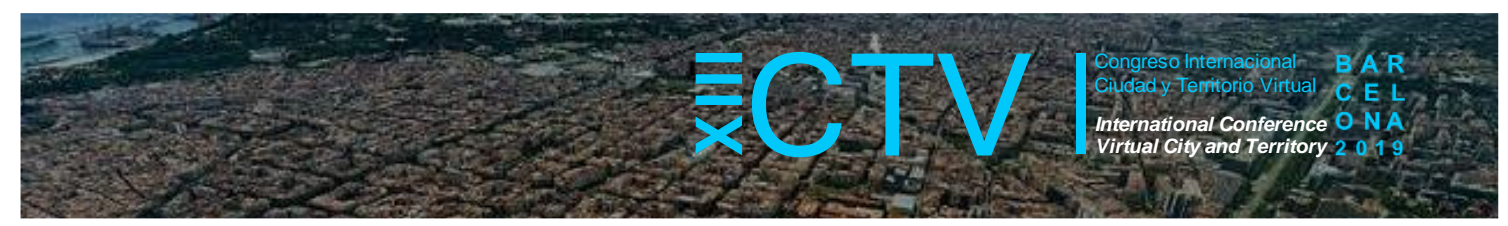

Figura 4. Representación conceptual del efecto de enfriamiento

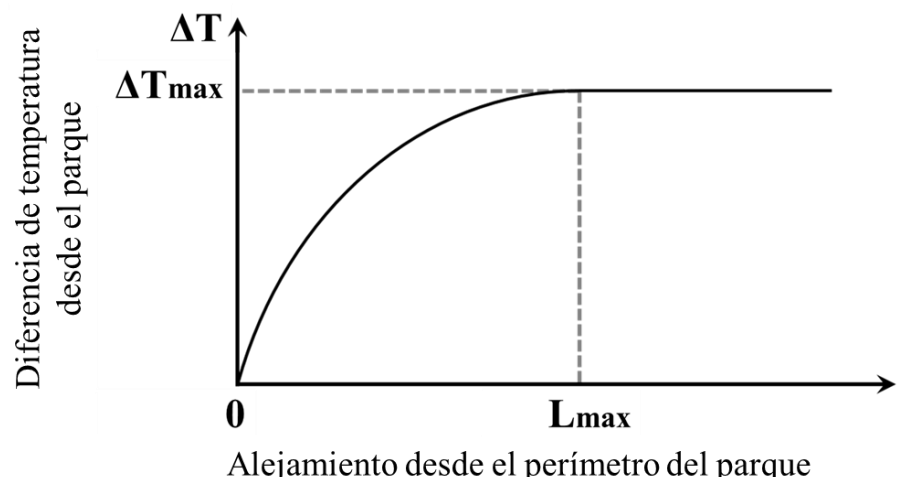

Fuente: Adaptada de Lin, Yu, Chang, Wu, y Zhang (2015).

El límite de extensión de enfriamiento (Lmax) es la distancia máxima alcanzada por la caída de temperatura ocasionada por un parque en el entorno urbano. El efecto de enfriamiento positivo implica temperaturas más bajas cerca del parque y un incremento gradual según más alejamiento. En un conjunto de datos de temperatura ajustada de espacios urbanos ordenados por distancia a un parque, el efecto de enfriamiento genera una "curva de enfriamiento", que termina en el $\Delta T$ máximo ( $\Delta T \max$ ) y define el punto Lmax (Figura 4), donde la temperatura alcanza la media de los alrededores más lejanos, comienza a disminuir o se estabiliza en una tendencia creciente (Lin, Yu, Chang, Wu y Zhang, 2015; Du, et al., 2017).

\subsection{Métodos analíticos}

\subsubsection{Delimitación de los alrededores mediante dos anillos concéntricos}

Como primer enfoque, se propone una delimitación de las áreas circundantes cercanas con dos anillos concéntricos en cada caso de estudio. El objetivo de esta división es cuantificar el $\Delta T$ de cada parque, en relación con su contexto particular y hacer un primer acercamiento para ubicar los rangos de distancia del Lmax. Primero, se generan dos anillos concéntricos de 0 a 100 metros (A1) y de 100 a $300 \mathrm{~m}$ (A2) de cada parque. En seguida, se calcula individualmente la LST media de cada anillo y parque excluyendo los píxeles de los otros parques dentro de los anillos. Además, se realiza una correlación lineal bivariada para evaluar la interacción entre las temperaturas de los parques y sus alrededores.

\subsubsection{Cuantificación mediante anillos concéntricos de 10m de ancho}

Como un enfoque más detallado, se proponen anillos concéntricos de $10 \mathrm{~m}$ de ancho para homogeneizar LST de los alrededores y cuantificar Lmax y $\Delta$ Tmax de cada parque. Adaptado de Du et al. (2017), dicho método permite identificar Lmax en todas las direcciones, teniendo en cuenta las disparidades causadas por el flujo del viento ( $\mathrm{Lu}$, Li, Liyue y Chen, 2017). Para esto, se crea un área concéntrica de $500 \mathrm{~m}$ de radio desde el perímetro de los parques y se divide en 50 anillos concéntricos de $10 \mathrm{~m}$ de ancho. Entonces, se calcula la LST media de cada parque y anillo excluyendo los píxeles de LST dentro de los otros parques. Por último, mediante un ajuste de curva polinómica de seis grados, se ajustan las fluctuaciones en cada conjunto de datos de 51 valores de LST (parque y 50 anillos), en los cuales se registra una correlación entre 0,71 y $0,95 R^{2}(p<0,01)$ con los valores originales. En las series ajustadas, se identifica la Lmax al final 


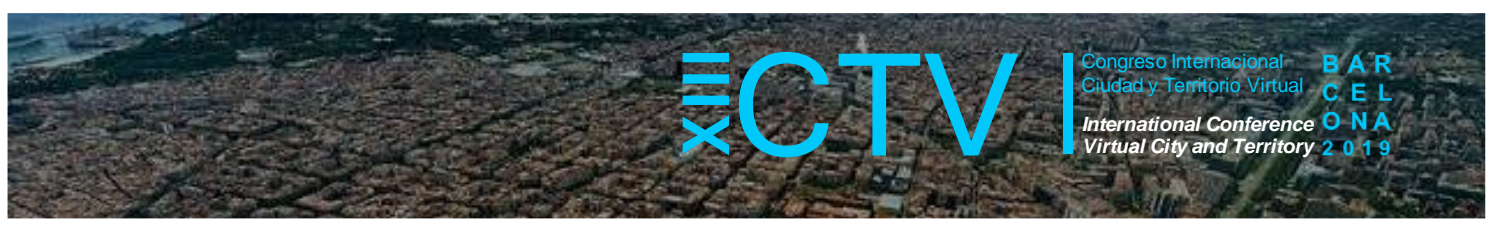

de la curva de enfriamiento y el $\Delta T \max$ se calcula como la diferencia entre la LST del anillo de la Lmax (como Tu) y la LST media del parque (como TP). En este método, se excluye la Rambla de Gavà debido a la estrechez de su sección. En cambio, se estudia con más detalle con el siguiente método.

\subsubsection{Cuantificación de variaciones de enfriamiento mediante secciones transversales}

Por último, se proponen secciones transversales para un análisis más detallado de las variaciones de $\Delta T \max$ y $L$ max de un parque dentro de diferentes contextos urbanos. Solo el caso de la Rambla de Gavà se aborda en esta etapa. Para complementar la división de 50 anillos concéntricos, se colocan secciones de $10 \mathrm{~m}$ de ancho transversales a la Rambla en toda su extensión. Resultando una cuadrícula de celdas de $10 \mathrm{~m}$ distribuidas en 59 filas que corresponden a las secciones transversales y 101 columnas que son los anillos divididos, 50 al noreste, 50 al suroeste y el correspondiente a la Rambla. Entonces, para calcular la LST media en dichas celdas, cada píxel de $30 \mathrm{~m}$ (Landsat-8) se divide en 36 sub-píxeles de $5 \mathrm{~m}$, los cuales conservan la LST del píxel. Donde se calcula la LST media de los sub-píxeles dentro de cada celda de $10 \mathrm{~m}$. En este caso, se crean dos conjuntos de 51 datos de LST para cada sección. Donde el primer valor corresponde a la Rambla en ambas series, y en uno los otros 50 al noreste y en el otro al suroeste. Por lo tanto, los cálculos de los indicadores de ajuste de curva y enfriamiento también se realizan en ambas orientaciones. Las secciones transversales de la Rambla se agrupan en tres zonas: 1) secciones en unidades industriales y comerciales; 2) secciones en el tejido urbano continuo; y 3) secciones adyacentes al Parc Torre Lluch.

\section{Resultados}

La totalidad del área de VGC registró $35.64^{\circ} \mathrm{C}$ de LST media. Mientras que las superficies artificiales por si solas registraron $36.69^{\circ} \mathrm{C}$ y las no artificiales $35.06^{\circ} \mathrm{C}$ (Figura 5). Particularmente, las superficies artificiales en los municipios registraron $35.41^{\circ} \mathrm{C}$ en Castelldefels, $37.15^{\circ} \mathrm{C}$ en Gavà y $38.02^{\circ} \mathrm{C}$ en Viladecans.

Figura 5. LST del 26 de Julio de 2018 en el área VGC

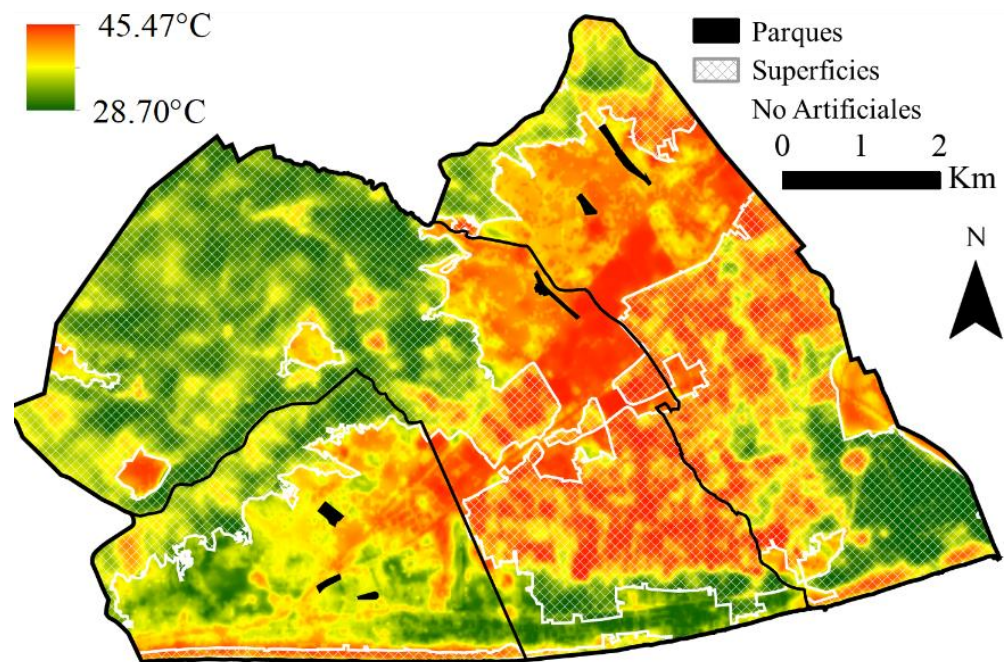

Fuente: Elaboración propia con información de USGS (2018), Copernicus Land Monitoring Service, (2016) y Barcelona Regional (2018) 


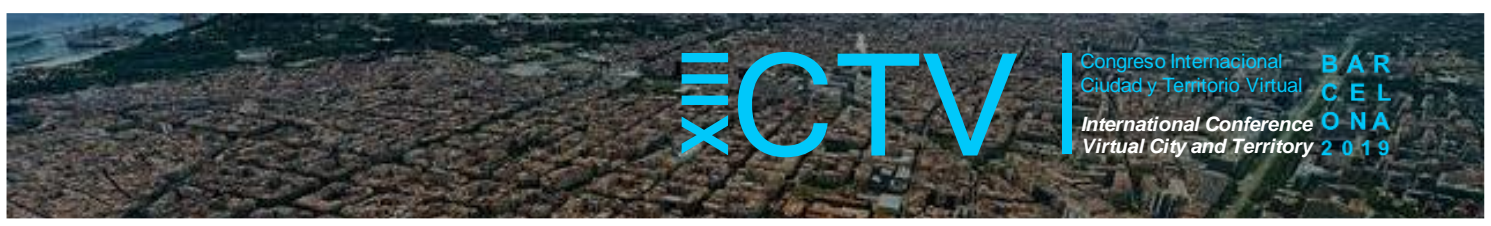

Las variaciones municipales también son apreciables en la LST de los parques, que tienden a disminuir al pasar de Viladecans a Castelldefels (Figura 6). Solo la Rambla de Gavà está fuera de esta tendencia, debido a sus características particulares de calle peatonal, así como su ubicación entre unidades industriales.

Figura 6. LST de los parques seleccionados

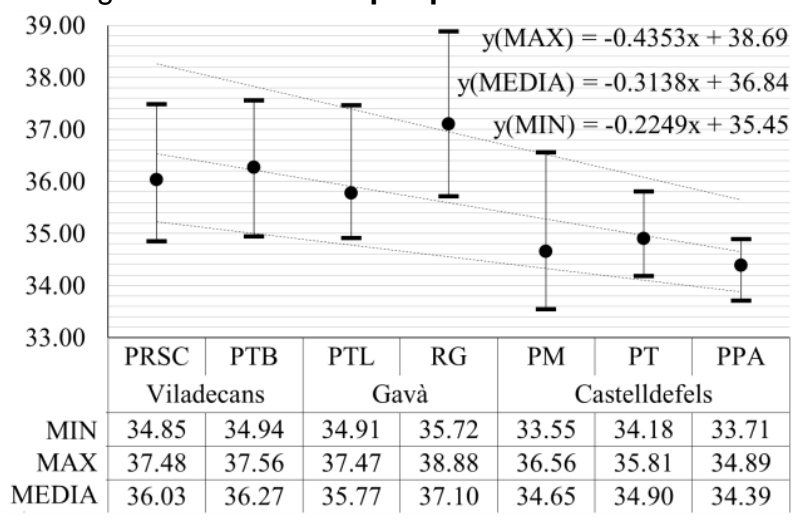

Fuente: Elaboración propia

En conjunto, los parques registraron una LST media de $35.61^{\circ} \mathrm{C}, 1.08^{\circ} \mathrm{C}$ más bajo que el de las superficies artificiales de la conurbación. Todos los parques resultaron en un LST más bajo que las superficies artificiales de sus respectivos municipios. Parc de la Riera de Sant Climent $1.99^{\circ} \mathrm{C}$, Parc Torrent Ballester $1.75^{\circ} \mathrm{C}$, Parc Torre Lluch $1.38^{\circ} \mathrm{C}$, Rambla de Gavà $0.05^{\circ} \mathrm{C}$, Parc de la Muntanyeta $0.76^{\circ} \mathrm{C}$, Parc dels Tellinaires $0.51^{\circ} \mathrm{C}$ y Parc de la Plaça d'Asturias registró $1.02^{\circ} \mathrm{C}$ más bajo que las superficies artificiales. En este punto, los parques en Viladecans registraron el mayor efecto de enfriamiento y aquellos en Castelldefels el más bajo.

\subsection{Efecto de enfriamiento en los alrededores más cercano}

Los resultados de la delimitación del entorno, confirman una reducción de temperatura en relación con el contexto urbano cercano de los parques. El conjunto de anillos de 0-100m (A1) y $100-300 \mathrm{~m}(\mathrm{~A} 2)$ de todos los parques, registraron $36.84^{\circ} \mathrm{C}$ y $37.08^{\circ} \mathrm{C}$ de LST media, lo que indica una reducción de $1.25^{\circ} \mathrm{C}$ y $1.50^{\circ} \mathrm{C}$ en relación con el entorno artificial. En los resultados particulares, los anillos resultaron en $\Delta$ Tmax positiva en todos los casos (Figura 7 ).

Figura 7. Anillos concéntricos a 100 y $300 \mathrm{~m}$ del perímetro de los parques y LST

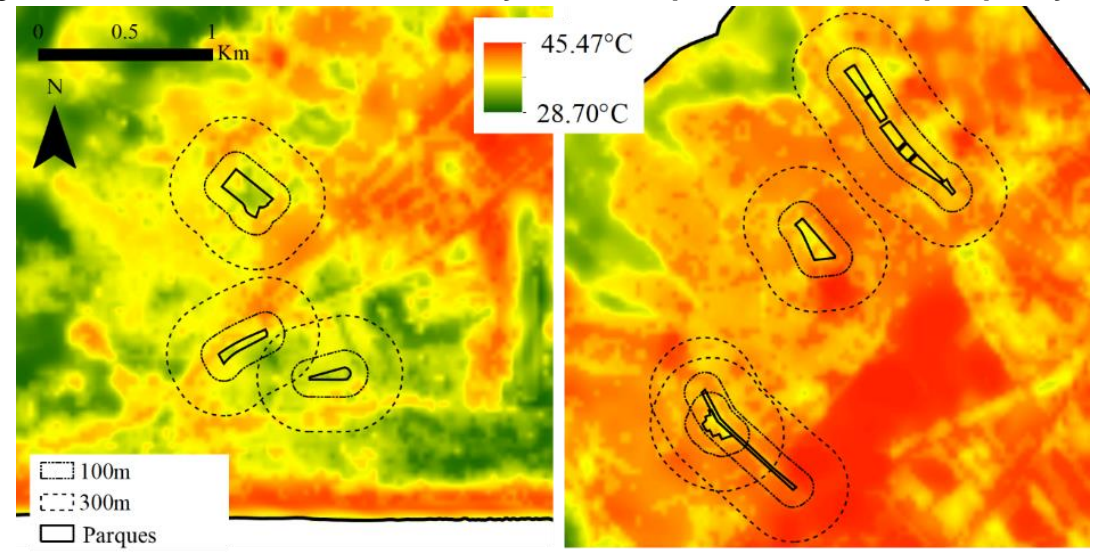

Fuente: Elaboración propia 


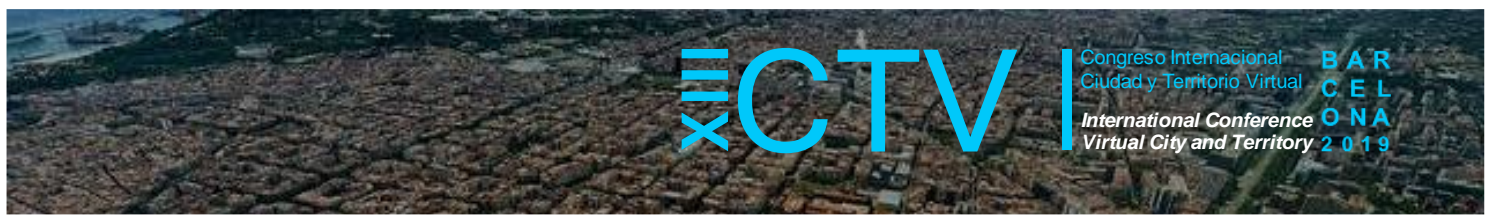

El anillo $A 1$ registró la $\Delta T \max$ más alta en el Parc Torre Lluch con $2.15^{\circ} \mathrm{C}$ y la más baja en el Parc de la Plaça d'Asturias con $0.66^{\circ} \mathrm{C}$. Así como el A2 registró su $\Delta$ Tmax más alta también en el Parc Torre Lluch con $2.22^{\circ} \mathrm{C}$ y la más baja en el Parc dels Tellinaires con $0.62^{\circ} \mathrm{C}$ (Tabla 1 ).

Tabla 1. LST de parques, anillos concéntricos e $\Delta$ Tmax

\begin{tabular}{|c|c|c|c|c|c|c|}
\hline Parque & $\begin{array}{c}\text { LST media } \\
\text { Parque }\end{array}$ & $\begin{array}{l}\text { LST media } \\
\mathbf{A}_{1}\end{array}$ & $\begin{array}{c}\text { LST media } \\
\mathrm{A}_{2}\end{array}$ & $\begin{array}{c}\Delta T \\
A_{1}-P\end{array}$ & $\begin{array}{c}\Delta T \\
A_{2}-P\end{array}$ & $\begin{array}{c}\Delta \mathrm{T} \\
\mathrm{A}_{2}-\mathrm{A}_{1}\end{array}$ \\
\hline PRSC & 36.03 & 37.12 & 37.55 & 1.09 & 1.52 & 0.43 \\
\hline PTB & 36.27 & 37.97 & 38.24 & 1.70 & 1.97 & 0.28 \\
\hline PTL & 35.77 & 37.92 & 37.99 & 2.15 & 2.22 & 0.07 \\
\hline $\mathbf{R G}$ & 37.10 & 38.36 & 39.07 & 1.26 & 1.96 & 0.71 \\
\hline PM & 34.65 & 35.82 & 36.18 & 1.17 & 1.53 & 0.36 \\
\hline PT & 34.90 & 35.61 & 35.52 & 0.71 & 0.62 & -0.08 \\
\hline PPA & 34.39 & 35.05 & 35.04 & 0.66 & 0.65 & -0.02 \\
\hline
\end{tabular}

Fuente: Elaboración propia

Cinco casos registraron su Lmax en el área A2. Los valores más altos de $\Delta T$ en este anillo implican un aumento prolongado de la temperatura más allá de los $100 \mathrm{~m}$ de distancia desde el parque (Figura 8). Sin embargo, el Parc dels Tellinaires y el Parc de la Plaça d'Asturias registran su $\Delta T$ máximo en el área $A 1$. Lo que implica que el efecto de enfriamiento de los parques no se extiende más allá del búfer de 100m desde los límites de los parques.

Figura 8. LST de parques y anillos concéntricos $A_{1}$ y $A_{2}$

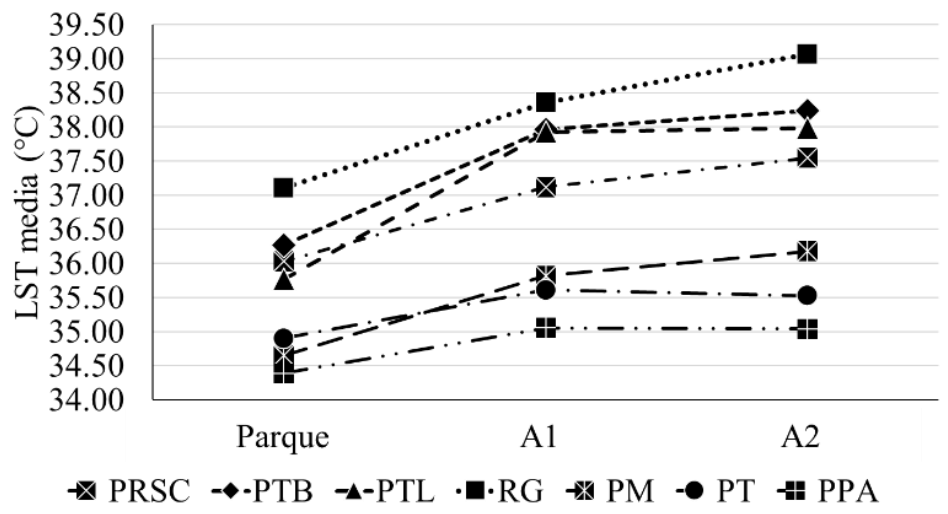

Fuente: Elaboración propia

En términos de ubicación, la LST de los contextos urbanos registró una mayor influencia en la $\Delta T$ que la propia LST de los parques. La influencia de la LST del área $A_{1}$ sobre el $\Delta T$ registró una correlación lineal de $0.59 \mathrm{R}^{2}(p<0.05)$ y la LST del anillo $\mathrm{A}_{2}$ registró una influencia de $0.81 \mathrm{R}^{2}$ $(p<0.01)$ (Figura 9). Mientras tanto, la LST de los parques resultó en una correlación no significativa $(p>0.05)$ con la $\Delta T$. Además, este comportamiento apunta a una mayor influencia del área del anillo $A_{2}$, mientras que por cada grado que aumenta su LST, la $\Delta T$ del parque aumenta en $0.3790^{\circ} \mathrm{C}$, y por cada grado que aumenta la LST del área más cercana, el $\Delta T$ aumenta $0.3067^{\circ} \mathrm{C}$.

Del mismo modo, esto está relacionado con la interacción de la LST media de los parques y la del anillo circundante, donde la LST de los parques registró una correlación lineal de $0.88 R^{2}$ $(p<0.01)$ con la LST del área $A_{1}$ y $0.92 R^{2}(p<0.01)$ con $A_{2}$. En general, cuanto mayor la temperatura del contexto urbano, mayor $\Delta T$ de los parques. 




Figura 9. Correlación entre la LST de los parques y la de los anillos concéntricos $A_{1}$ y $A_{2}$
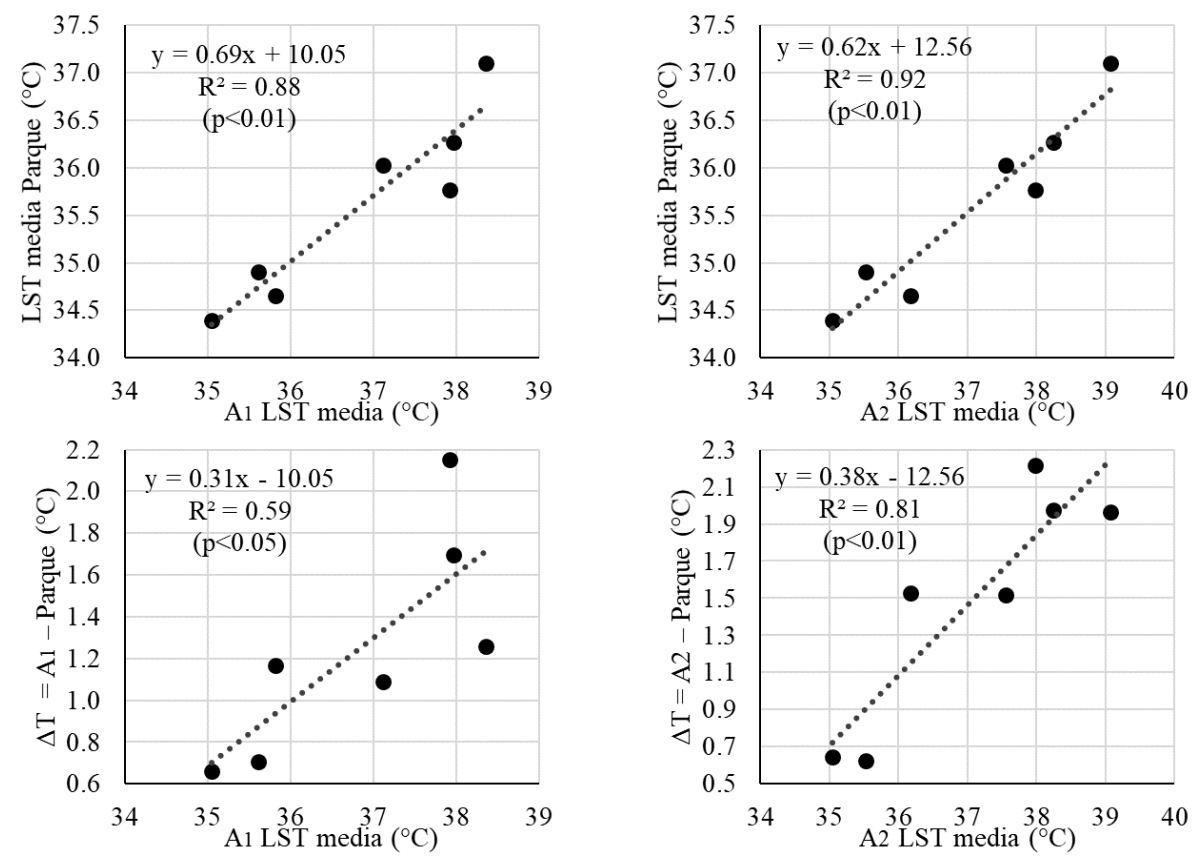

Fuente: Elaboración propia

\subsection{Efecto de enfriamiento cuantificado con anillos concéntricos de $10 \mathrm{~m}$ de ancho}

La cuantificación del enfriamiento por anillos concéntricos de $10 \mathrm{~m}$ de ancho registró un efecto positivo en los seis parques evaluados en esta etapa. Los parques resultaron en un promedio de $91.67 \mathrm{~m}$ de Lmax y $1.22^{\circ} \mathrm{C}$ de $\Delta \mathrm{T}$ (Tabla 2). La Lmax máxima corresponde al Parc Torrent Ballester con $130 \mathrm{~m}$ y la mínima al Parc de la Plaça d'Asturias con $40 \mathrm{~m}$. La $\Delta T$ max máxima resultó en $2.16^{\circ} \mathrm{C}$ y mínimo de $0.29^{\circ} \mathrm{C}$, correspondiente a ambos últimos.

Tabla 2. Indicadores de enfriamiento obtenidos mediante anillos concéntricos de 10m de ancho

\begin{tabular}{|c|c|c|c|c|c|}
\hline Parques & $\begin{array}{c}\text { LST } \\
\text { parque }\left({ }^{\circ} \mathbf{C}\right)\end{array}$ & $\begin{array}{c}\text { LST } \\
\text { ajustada } \\
\text { parque }\left({ }^{\circ} \mathbf{C}\right)\end{array}$ & $\begin{array}{c}\text { LST } \\
\text { ajustada } \\
L_{\max }\left({ }^{\circ} \mathbf{C}\right)\end{array}$ & $L_{\max }(m)$ & $\Delta \mathrm{T}\left({ }^{\circ} \mathrm{C}\right)$ \\
\hline PRSC & 36.03 & 36.17 & 37.19 & 80 & 1.02 \\
\hline PTB & 36.27 & 36.26 & 38.42 & 130 & 2.16 \\
\hline$\overline{\text { PTL }}$ & 35.77 & 35.74 & 37.73 & 100 & 1.99 \\
\hline $\mathbf{P M}$ & 34.65 & 34.85 & 36.08 & 120 & 1.24 \\
\hline PT & 34.90 & 35.15 & 35.76 & 80 & 0.61 \\
\hline PPA & 34.39 & 34.71 & 35.00 & 40 & 0.29 \\
\hline Avg & 35.61 & 35.48 & 36.70 & 91.67 & 1.22 \\
\hline
\end{tabular}

Fuente: Elaboración propia

En Viladecans, el Parc de la Riera de Sant Climent registró su Lmáx a $80 \mathrm{~m}$ y $1.02^{\circ} \mathrm{C}$ de $\Delta \mathrm{T}$, pero la LST máxima se alcanza a $250 \mathrm{~m}$ del parque con $1.47^{\circ} \mathrm{C}$ de $\Delta T$. Variaciones relacionadas con las áreas rurales adyacentes y el tejido urbano discontinuo hacia el este, con temperaturas más bajas que el tejido urbano continuo hacia el oeste. Mientras tanto, el Parc Torrent Ballester, registró una curva de enfriamiento clara hasta la Lmáx de $130 \mathrm{~m}$ y una $\Delta T$ de $2.16^{\circ} \mathrm{C}$, seguido de una caída de temperatura en áreas urbanas más alejadas. En este caso, la LST más alta se concentra en los alrededores más cercanos porque el parque está rodeado por un tejido urbano continuo. 


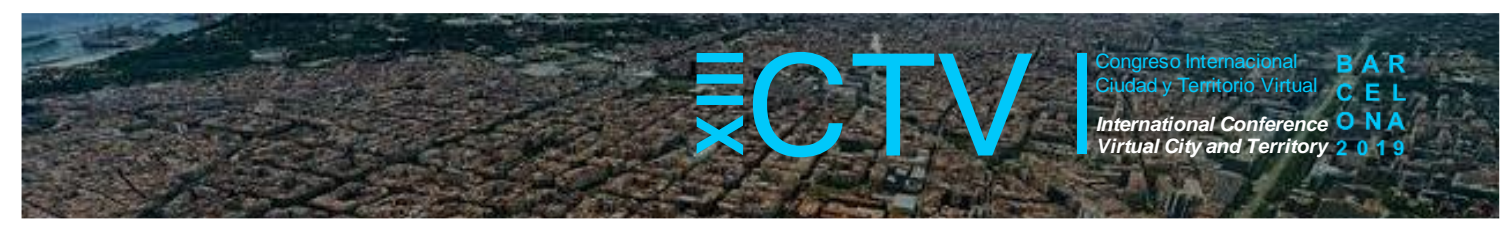

En Gavà, el Parc Torre Lluch registra una Lmax de $100 \mathrm{~m}$ y $1.99^{\circ} \mathrm{C}$ de $\Delta \mathrm{T}$, seguido de temperaturas crecientes en las áreas urbanas más alejadas. Comportamiento relacionado con el contacto directo con la Rambla de Gavà (estudiado en la próxima sección), que genera un efecto conjunto en el tejido urbano discontinuo.

Castelldefels registró diferencias importantes en sus parques. El Parc de la Muntanyeta, rodeado de áreas verdes urbanas y tejido urbano discontinuo, alcanzó una Lmax de $120 \mathrm{~m}$ con una $\Delta T$ de $1.24^{\circ} \mathrm{C}$, con una LST en aumento en áreas urbanas más alejadas. En el Parc dels Tellinaires, el ferrocarril y la carretera adyacentes hacia el norte causan incrementos de temperatura en sus alrededores más cercanos, que contienen el Lmax a $80 \mathrm{~m}$ y $0.61^{\circ} \mathrm{C}$ de $\Delta \mathrm{T}$. Mientras tanto, el Parc de la Plaça d'Asturias contenido dentro de un desarrollo de viviendas en su perímetro, registró un Lmax de $40 \mathrm{~m}$ con una $\Delta T$ de $0.29^{\circ} \mathrm{C}$, seguidos por valores de LST en descenso que comienzan a aumentar a $100 \mathrm{~m}$ de distancia hasta un segundo pico de temperatura en el $200 \mathrm{~m}$.

La comparación de los seis parques señala la influencia de su entorno en su efecto de enfriamiento (Figura 10). En particular, la LST media de los parques que registraron un rango de $1.88^{\circ} \mathrm{C}$ entre un mínimo de $34.36^{\circ} \mathrm{C}$ en el Parc de la Plaça d'Asturias y un máximo de $36.27^{\circ} \mathrm{C}$ en el Parc Torrent Ballester. En coincidencia con el rango de $3.42^{\circ} \mathrm{C}$ del LST de los puntos Lmax, calculado también con los dos últimos parques como mínimo y máximo, respectivamente.

Figura 10. Series de LST ajustadas (LST Parque $=\mathbf{0}$ ) y cálculo de indicadores de enfriamiento


Distancia al perímetro del parque

Distancia al perímetro del parque

Parque $\quad$ Serie ajustada de LST

Lmax $\Delta \operatorname{Tmax}$

Fuente: Elaboración propia

\subsection{Efecto de enfriamiento cuantificado con anillos concéntricos de $10 \mathrm{~m}$ de ancho}

La totalidad de Rambla de Gavà registró una LST media absoluta de $37,10^{\circ} \mathrm{C}$ y el área evaluaa mediante las secciones transversales registró una LST media de $36,94^{\circ} \mathrm{C}$, ambos valores más altos que el resto de parques. 


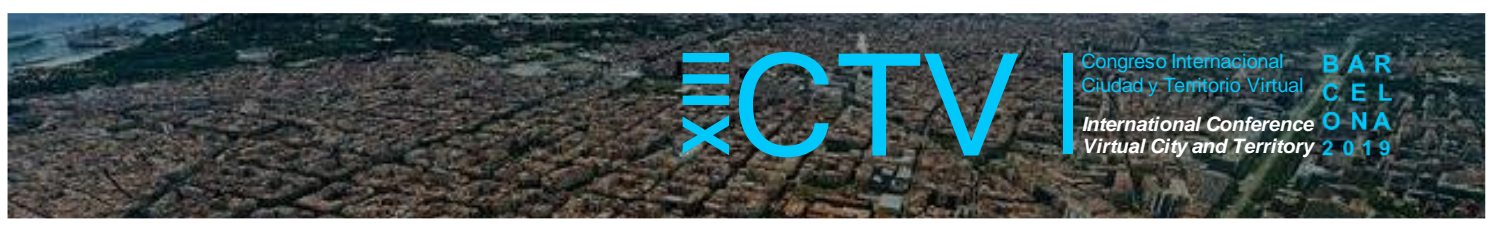

Figura 11. Malla de $10 \mathrm{~m}$ con anillos concéntricos y secciones transversales en Rambla

Fuente: Elaboración propia

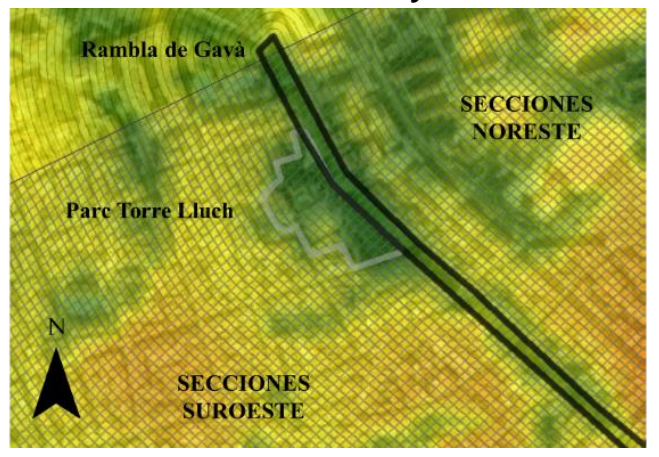

La Rambla dividida por los diferentes contextos urbanos (Figura 12), dio como resultado una LST media de $38.22^{\circ} \mathrm{C}$ en las unidades industriales, $37.24^{\circ} \mathrm{C}$ en el tejido urbano y $35.72^{\circ} \mathrm{C}$ en la sección en contacto con el Parc Torre Lluch. Estos valores muestran una reducción de la temperatura de $2,21^{\circ} \mathrm{C}, 1,05^{\circ} \mathrm{C}$ y $1,76^{\circ} \mathrm{C}$ respectivamente, en relación con la LST media del entorno urbano dentro de un área a $300 \mathrm{~m}$ de la Rambla (Figura 13).

Figura 12. LST en malla de 10m en los alrededores de la Rambla: a) representación de LST media por celda; b) LST ajustada por sección en ambos sentidos (noreste y suroeste) de la Rambla; y c) relieve de LST ajustada por sección

a)

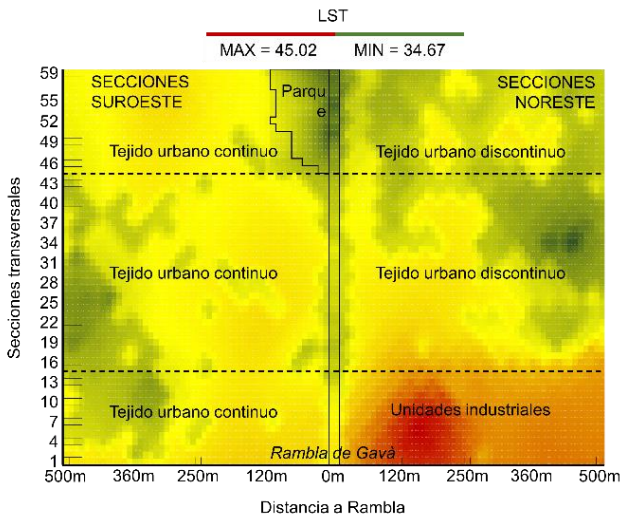

b)

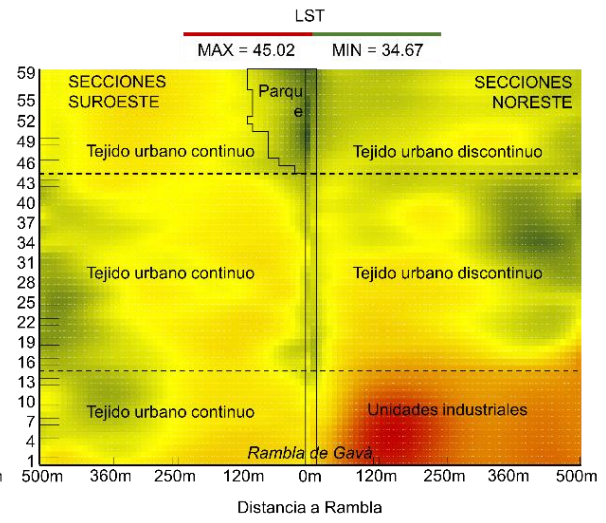

c)

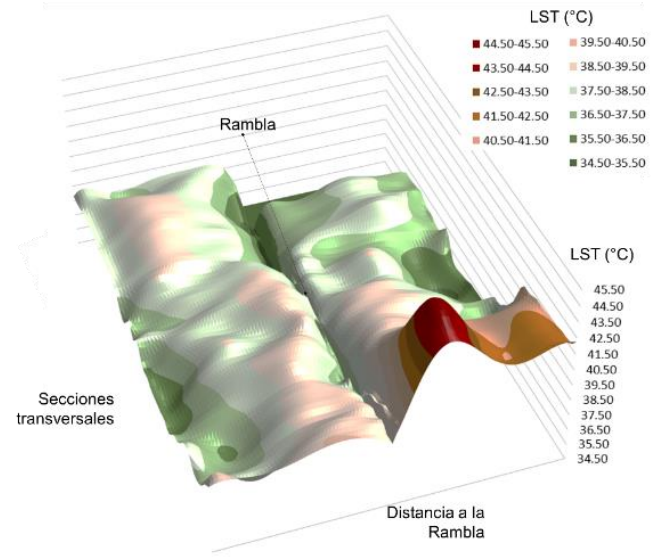

Fuente: Elaboración propia 
Figura 13. LST media de secciones transversales por contexto urbano

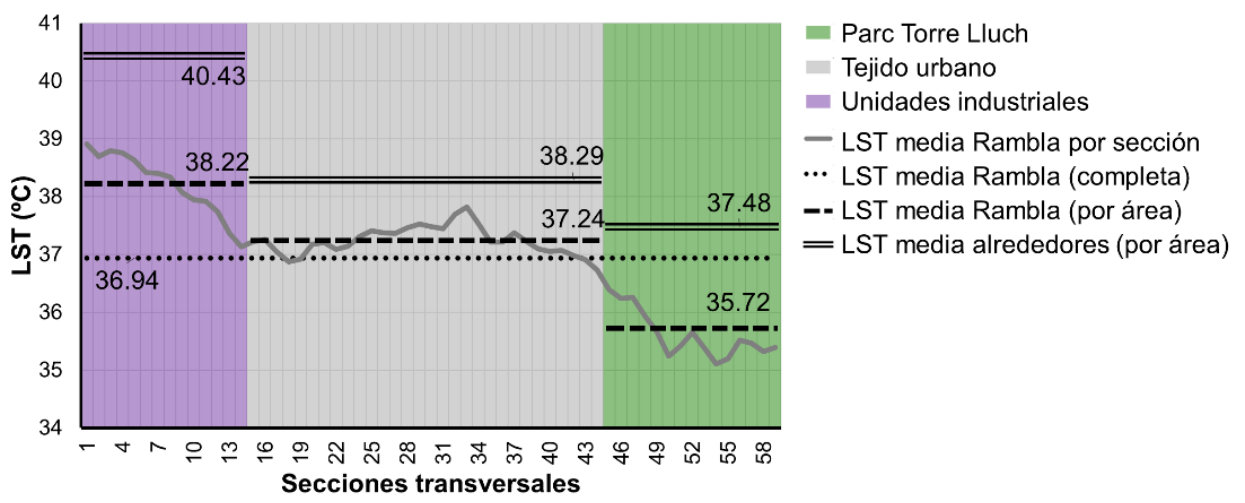

Fuente: Elaboración propia

En términos de orientación, la Rambla registró variaciones de $\Delta T$ en relación con la LST media de los alrededores. Al norte y al sur en la zona de unidades industriales, registraron $3.91^{\circ} \mathrm{C} \mathrm{y}$ $0.51^{\circ} \mathrm{C}$ respectivamente. En el área adjunta al Parc Torre Lluch se registraron $\Delta \mathrm{T}$ de $1.48^{\circ} \mathrm{C}$ al norte y $2.03^{\circ} \mathrm{C}$ al sur. Mientras tanto, el área del tejido urbano registró una diferencia menor, con $1.16^{\circ} \mathrm{C}$ respecto al tejido urbano discontinuo al norte y $1.09^{\circ} \mathrm{C}$ al continuo hacia el sur.

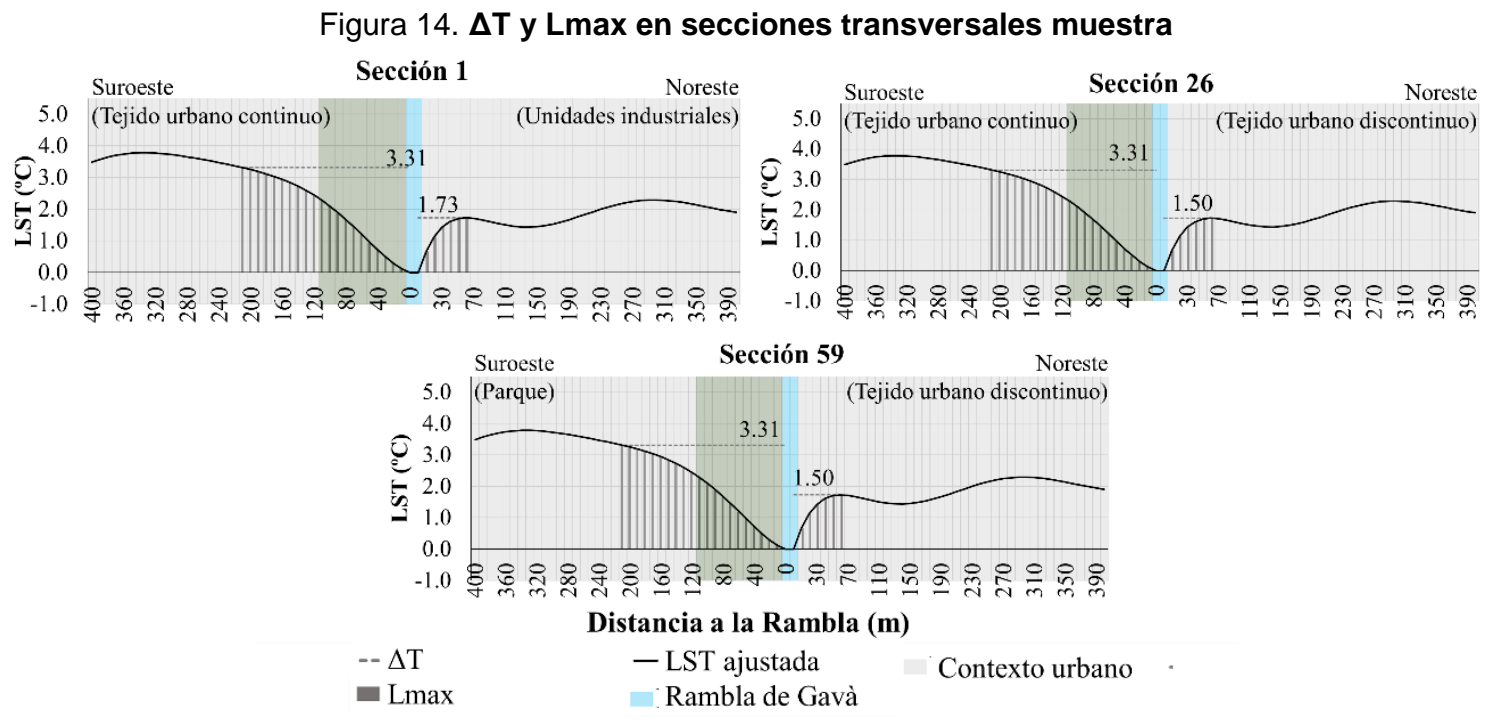

Fuente: Elaboración propia

El cálculo de Lmax para cada sección transversal coincidió con la mayor diferencia por orientación en el área industrial. La sección 1 (Figura 14) registró una Lmax de $140 \mathrm{~m}$ y $4.81^{\circ} \mathrm{C}$ de $\Delta T$ en relación con las secciones norte con unidades industriales, mientras que en la sección sur con tejido urbano continuo la Lmax alcanzó $150 \mathrm{~m}$ pero con $\Delta \mathrm{T}$ de $0.95^{\circ} \mathrm{C}$. Al igual que en la sección 8 , más cerca del tejido urbano, resultó en un Lmáx de $150 \mathrm{~m}$ y $5.71^{\circ} \mathrm{C} \Delta \mathrm{T}$ al norte, mientras que al sur solo alcanzó $40 \mathrm{~m}$ y $0.38^{\circ} \mathrm{C}$ de $\Delta \mathrm{T}$. En el centro del tejido urbano, la sección 26 (Figura 14) registró una Lmáx de $170 \mathrm{~m}$ con $1.75^{\circ} \mathrm{C}$ de $\Delta \mathrm{T}$ al norte con tejido urbano discontinuo, y $60 \mathrm{~m}$ de $\mathrm{Lmax}$ y $1.64^{\circ} \mathrm{C}$ de $\Delta \mathrm{T}$ al sur con tejido urbano continuo. Del mismo modo, en la sección 44, en el límite entre el tejido urbano y el Parc Torre Lluch, la Lmáx al norte alcanzó los $50 \mathrm{~m}$ y $0.75^{\circ} \mathrm{C}$ de $\Delta \mathrm{T}$, mientras que al sur alcanzó los $180 \mathrm{~m}$ y $1.54^{\circ} \mathrm{C}$ de $\Delta \mathrm{T}$. 


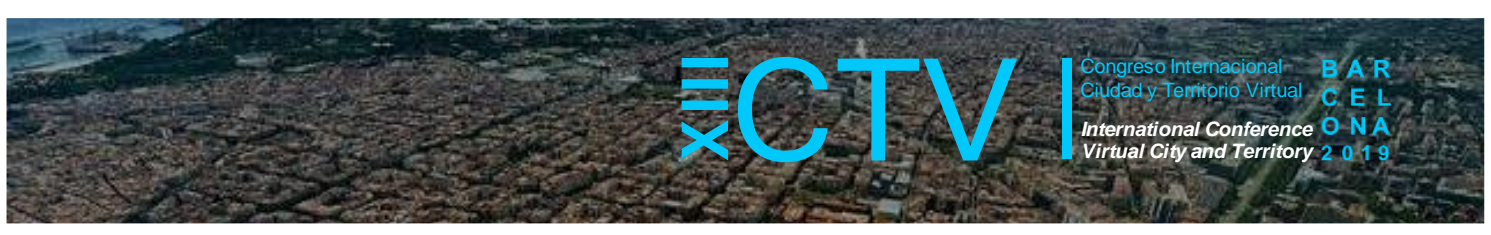

Luego, en la sección 59 (Figura 14) en el área adyacente al parque, se registra una Lmáx de $80 \mathrm{~m}$ y $1.53^{\circ} \mathrm{C}$ de $\Delta \mathrm{T}$ al norte. Mientras tanto, hacia el sur, incluso cuando la Rambla registra la LST media más baja, la serie LST ajustada en esta orientación muestra variaciones debido al efecto conjunto de la Rambla y el Parc Torre Lluch. Se observa un primer punto de ruptura a $40 \mathrm{~m}$ de la Rambla con $0.74^{\circ} \mathrm{C}$ de $\Delta T$, donde la tendencia cambia. El segundo punto de ruptura se registra en los $190 \mathrm{~m}$ de distancia de la Rambla con $2.96^{\circ} \mathrm{C}$ de $\Delta \mathrm{T}$, donde la tendencia de la curva de enfriamiento se vuelve constante y aumenta hasta alcanzar el tercer punto de ruptura, en los $330 \mathrm{~m}$ con LST $3.60^{\circ} \mathrm{C}$ más alta que la de la Rambla.

\section{Figura 15. Lmax a lo largo de todas las secciones transversales}

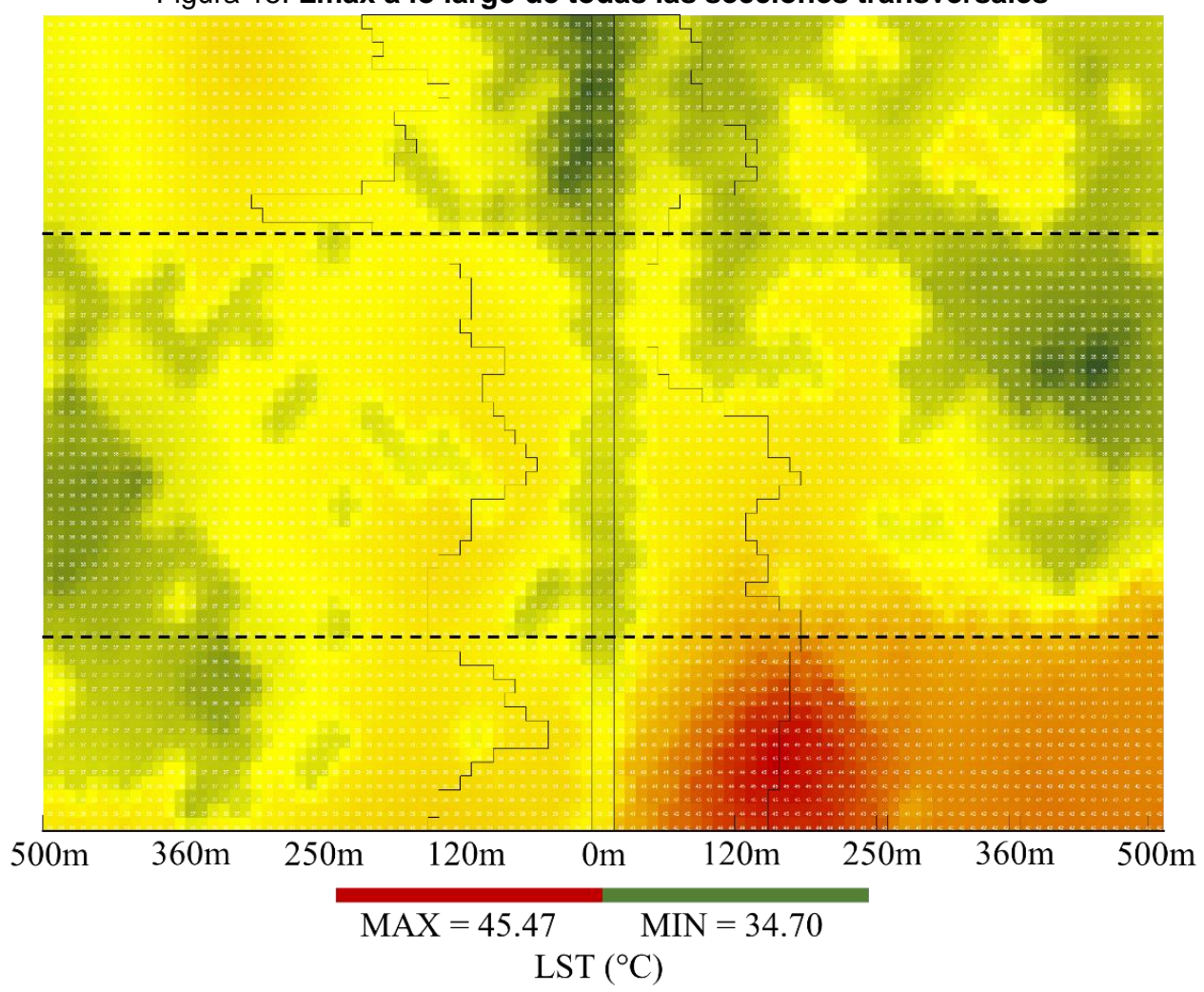

Fuente: Elaboración propia

Por último, la cuantificación del efecto de enfriamiento de todas las secciones en ambas orientaciones da como resultado una delimitación espacial detallada del Lmax en toda la Rambla (Figura 17). La Lmax promedio alcanzó $109.00 \mathrm{~m}$ hacia el noreste con $170 \mathrm{~m}$ como máximo en las unidades industriales y 30m como mínimo en el tejido urbano, así como $129.67 \mathrm{~m}$ de promedio hacia el suroeste con $310 \mathrm{~m}$ como máximo en el área con el parque y 40m como mínimo en el tejido urbano.

\section{Conclusiones}

Primero, las conclusiones sobre los métodos aplicados se resumen como sigue:

- Los tres métodos aplicados registraron un potencial de enfriamiento de los parques semejante. Este análisis de múltiples etapas señala que los métodos analíticos 


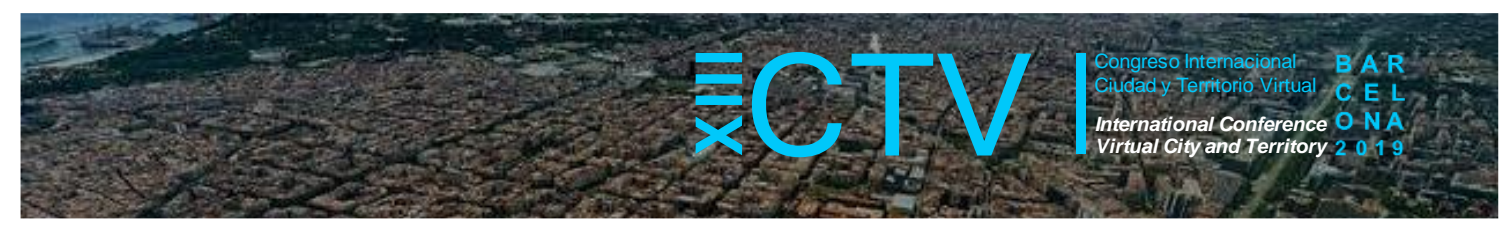

espaciales para medir las variaciones de temperatura en las ciudades son adecuados para reconocer la influencia de la composición física del entorno construido. Aunque, este estudio solo se acerca a la clasificación de la cobertura del suelo como atributos físicos, los resultados en las múltiples escalas de detalle, señalaron la misma interacción del efecto de enfriamiento con la forma urbana.

- Los tres métodos son adecuados para análisis de mayor escala, pero la delimitación de los espacios a través de dos anillos concéntricos se considera la más adecuada debido a la cantidad de datos obtenidos de los otros dos métodos analíticos. Los resultados en la primera etapa representan un marco general de los resultados de las otras etapas. En particular, la delimitación a través de los dos anillos permite el análisis generalizado del efecto de enfriamiento de los espacios verdes considerando mayores porciones de territorio.

- La extensión de enfriamiento a través de la sección transversal se considera la más adecuada para cuantificar la influencia de la composición del entorno construido. Como se muestra en los resultados, este método da como resultado una imagen detallada del efecto de enfriamiento. Sin embargo, este método exige varios pasos de clasificación espacial para analizar los datos resultantes, y se limita a casos particulares de estudio.

Las conclusiones sobre la influencia del entorno construido son las siguientes:

- El entorno urbano de los parques registró máyor influencia en el efecto de enfriamiento que la LST de los parques. Particularmente en esta área de estudio, el efecto de enfriamiento resultó más relacionado con las variaciones de la temperatura de las áreas circundantes. Se considera necesaria más investigación para explorar este tema.

- Cuanto mayor es la temperatura del contexto urbano, mayor es la intensidad del efecto de enfriamiento. Sin embargo, la LST más alta en los espacios urbanos más cercanos causa un efecto de enfriamiento reducido. Esta afirmación está relacionada con la contención del efecto de enfriamiento causado por los densos espacios construidos cerca del parque. En estos casos, los parques no pueden compensar el calor en sus alrededores y su efecto de enfriamiento se ve limitado. Se necesita más investigación con el objetivo de ponderar el papel de los parques y la composición de su entorno.

Por último, los cambios de cubiertas de suelo se relaciona con las variaciones de temperatura del contexto urbano y por lo tanto en los indicadores del efecto de enfriamiento. Donde las temperaturas más altas en las unidades industriales, resultaron en una mayor intensidad de enfriamiento. Sin embargo, en el caso de la Rambla, el tejido urbano continuo causó un efecto de enfriamiento menor que el discontinuo, lo que está relacionado con el detalle del análisis que refleja las singularidades de espacios particulares.

Agradecimientos: El presente estudio es parte del proyecto "Urban-CLIMPLAN. La isla de calor urbana: efectos en el cambio climático y modelado para estrategias de planeamiento territorial y urbano. Aplicación a la región metropolitana de Barcelona" (Ref. BIA2015-68623-R). Financiado por el Ministerio de Economía y Competitividad de España (MINECO) y el Fondo Europeo para el Desarrollo Regional (FEDER).

Conflicto de Intereses: Los autores declaran que no hay conflicto de intereses. 


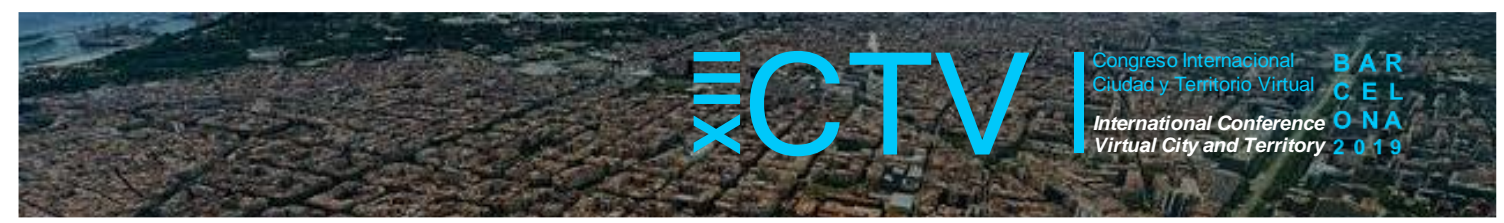

\section{Referencias}

Anjos, M., \& Lopes, A. (2017). Urban Heat Island and Park Cool Island Intensities in the Coastal City of Aracaju, North-Eastern Brazil. Sustainability (9), 1379 . doi: https://doi.org/10.3390/su9081379

Arbuthnott, K. G., \& Hajat, S. (2017). The health effects of hotter summers and heat waves in the population of the United Kingdom: a review of the evidence. Environmental Health, 16(1). doi: https://doi.org/10.1186/s12940-017-0322-5

Arellano, B., \& Roca, J. (2016). Identifying urban heat island: The Barcelona case. 11th International Congress form the cycle "Ciudad y Territorio Virtual" CTV (pp. 798-812). Cracovia: Centre de Política de Sòl i Valoracions. doi: http://upcommons.upc.edu/handle/2117/90350

Artis, D. A., \& Carnahan, W. H. (1982). Survey of emissivity variability in thermography of urban areas. Remote Sensing of Environment, 12(4), 313-329. doi: https://doi.org/10.1016/00344257(82)90043-8

Barcelona Regional. (2018, Julio 22). Parcs Seleccionats. Cirteris per l'estudi del comportament climàtic del continu urbà Viladecans-Gavà-Castelldefels. Barcelona, Spain.

Barsi, J., Schott, J., Hook, S., Raqueno, N., Markham, B., \& Radocinski, R. (2014). Landsat-8 Thermal Infrared Sensor (TIRS) Vicarious Radiometric Calibration. Remote Sensing, 6(11), 11607-11626. doi: https://doi.org/10.3390/rs61111607

Bowler, D., Buyung-Ali, L., Knight, T., \& Pullin, A. (2010). Urban greening to cool towns and cities: A systematic review of the empirical evidence. Landscape and Urban Planning, 147-155. doi: https://doi.org/10.1016/j.landurbplan.2010.05.006

Cao, X., Onishi, A., Chen, J., \& Imura, H. (2010). Quantifying the cool island intensity of urban parks using ASTER and IKONOS data. Landscape and Urban Planning, 96, 224-231. doi: https://doi.org/10.1016/j.landurbplan.2010.03.008

Carreras, C., Marín, M., Martín-Vide, J., Moreno, M. C., \& Sabí, J. (1990). Modificaciones térmicas en las ciudades. Avance sobre la isla de calor en Barcelona. Documents d'Anàlisi Geogràfica (17), 51-77. doi: http://hdl.handle.net/2445/22624

Cheng, X., Wei, B., Chen, G., Li, J., \& Song, C. (2015). Influence of Park Size and Its Surrounding Urban Landscape Patterns on the Park Cooling Effect. Journal of Urban Planning and Development, 141(3). doi: https://doi.org/10.1061/(ASCE)UP.1943-5444.0000256

Chow, W., Pope, R., Martin, C., \& Brazel, A. (2011). Observing and modeling the nocturnal park cool island of an arid city: horizontal and vertical impacts. Theoretical and Applied Climatology, 103(1-2), 197-211. doi: https://doi.org/10.1007/s00704-010-0293-8

Copernicus Land Monitoring Service. (2016, Septiembre 19). Corine Land Cover 2012 Version 18. Recuperado de Copernicus Land Monitoring Service: https://land.copernicus.eu/paneuropean/corine-land-cover/clc-2012

Declet-Barreto, J., Brazel, A. J., Martin, C., \& Chow, W. T. (2013). Creating the park cool island in an inner-city neighborhood: heat mitigation strategy for Phoenix, AZ. Urban Ecosystems, 16(3), 617-635. doi: https://doi.org/10.1007/s11252-012-0278-8 


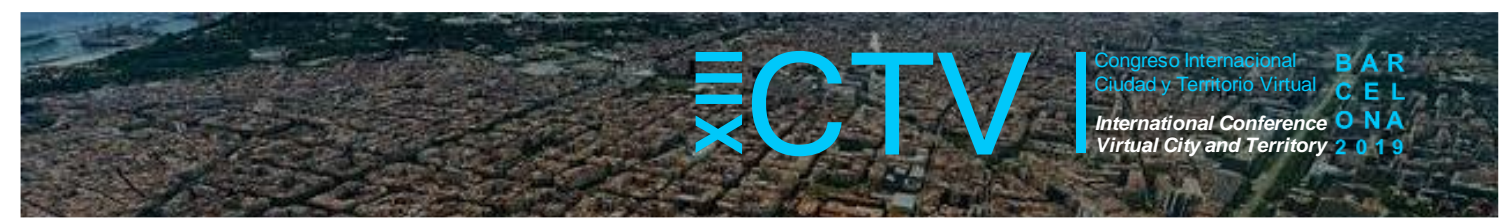

Du, H., Cai, W., Xu, Y., Wang, Z., Wang, Y., \& Cai, Y. (2017). Quantifying the cool island effects of urban green spaces using remote sensing Data. Urban Forestry \& Urban Greening (27), 2431. doi: http://dx.doi.org/10.1016/j.ufug.2017.06.008

Esri. (2019, Enero 10). World Imagery [basemap]. World Imagery Map of the Conurbation of Vialdecans, Gavà and Castelldefels, Spain. Esri, DigitalGlobe, GeoEye, i-cubed, USDA FSA, USGS, AEX, Getmapping, Aerogrid, IGN, IGP, swisstopo, and the GIS User Community. $\begin{array}{llllll}\text { Recuperado el } & 22 \text { de } & 2019 & \text { de }\end{array}$ http://www.arcgis.com/home/item.html?id=10df2279f9684e4a9f6a7f08febac2a9

Feyisa, G., Dons, K., \& Meilby, H. (2014). Efficiency of parks in mitigating urban heat island effect: An example from Addis Ababa. Landscape and Urban Planning, 87-95. doi: http://dx.doi.org/10.1016/j.landurbplan.2013.12.008

Hamada, S., Tanaka, T., \& Ohta, T. (2013). Impacts of land use and topography on the cooling effect of green areas on surrounding urban areas. Urban Forestry \& Urban Greening, 426-434. doi: https://doi.org/10.1016/j.ufug.2013.06.008

Hathway, E., \& Sharples, S. (2012). The interaction of rivers and urban form in mitigating the Urban Heat Island effect: A UK case study. Building and Environment, 58, 14-22. doi: https://doi.org/10.1016/j.buildenv.2012.06.013

Jauregui, E. (1990). Influence of a Large Urban Park on Temperatures and Convective Precipitation in a Tropical City. Energy and Buildings, 15-16(3-4), 457-463. doi: https://doi.org/10.1016/0378-7788(90)90021-A

Kuttler, W. (2012). Climate Change on the Urban Scale - Effects and Counter-Measures in Central Europe. In N. Chetri (Ed.), Human and Social Dimensions of Climate Change (pp. 105142). Rijeka, Croatia: InTech. doi: http://dx.doi.org/10.5772/50867

Lin, W., Yu, T., Chang, X., Wu, W., \& Zhang, Y. (2015). Calculating cooling extents of green parks using remote sensing: Method and test. Landscape and Urban Planning, 66-75. doi: https://doi.org/10.1016/j.landurbplan.2014.10.012

Lu, J., Li, Q., Liyue, Z., \& Chen, J. (2017). A micro-climatic study on cooling effect of an urban park in a hot and humid climate. Sustainable Cities and Society, 32, 513-522. doi: http://dx.doi.org/10.1016/j.scs.2017.04.017

Martin-Vide, J., Cordobilla, M., Moreno, M. C., \& Montlleó, M. (2015). La isla de calor en el área metropolitana de Barcelona y la adaptación al cambio climático. Àrea Metroplitana de Barcelona, Direcció de Serveis Ambientals de I'AMB. Barcelona Regional. Barcelona: Observatorio Metropolitano del Cambio Climático. Recuperado de http://www.amb.cat/es/web/mediambient/actualitat/publicacions/detall/-/publicacio/la-isla-de-calor-en-el-area-metropolitana-debarcelona-y-la-adaptacion/5773152/11818

Norton, B., Coutts, A., Livesley, S., Harris, R., Hunter, A., \& Williams, N. (2015). Planning for cooler cities: A framework to prioritise green infraestructure to mitigate high temperatures in urban landscapes. Landscape and Urban Planning, 127-138. doi: http://dx.doi.org/10.1016/j.landurbplan.2014.10.018

Oke, T. (1982). The energetic basis of the urban heat island. Quarterly Journal of the Royal Meteorological Society (108), 1-24. doi: http://dx.doi.org/10.1002/qj.49710845502

Patz, J. A., Campbell-Lendrum, D., Holloway, T., \& Foley, J. A. (2005). Impact of regional climate change on human health. Nature, 438, 310-317. doi: https://doi.org/10.1038/nature04188 




Ren, Z., He, X., Zheng, H., Zhang, D., Yu, X., Shen, G., \& Guo, R. (2013). Estimation of the Relationship between Urban Park Characteristics and Park Cool Island Intensity by Remote Sensing Data and Field Measurement. Forests, 4(4), 868-886. doi: https://doi.org/10.3390/\$4040868

Roca, J., Arellano, B., \& Batlle, E. (2018). Green areas and urban heat island: combining remote sensed data with ground observations. Remote Sensing and Modeling of Ecosystems for Sustainability XV, SPIE Proceedings 10767. San Diego, California, United States. doi: https://doi.org/10.1117/12.2320999

Sobrino, J., Jiménez-Muñoz, J., \& Paolini, L. (2004). Land surface temperature retrieval from LANDSAT TM 5. Remote Sensing of Environment, 90(4), 434-440. doi: http://doi.org/10.1016/j.rse.2004.02.003

Spronken-Smith, R., \& Oke, T. (1998). The thermal regime of urban parks in two cities with different summer climates. International Journal of Remote Sensing, XIX (11), 2085-2104. doi: http://dx.doi.org/10.1080/014311698214884

Stathopoulou, M., \& Cartalis, C. (2007). Daytime urban heat islands from Landsat ETM+ and Corine land cover data: An application to major cities in Greece. Solar Energy, 358-368. doi: https://doi.org/10.1016/j.solener.2006.06.014

U.S. Geological Survey (USGS). (2018). Image of Metropolitan Area of Barcelona, Spain at July 26 of 2018. Landsat-8 OLI/TIRS imagery. Recuperado de https://earthexplorer.usgs.gov/

U.S. Geological Survey. (2016, Marzo 26). Landsat 8 Data Users Handbook Version 2.0. Recuperado de Landsat missions. U.S. Geological Survey: https://landsat.usgs.gov/

Yan, H., Wu, F., \& Dong, L. (2018). Influence of a large urban park on the local urban thermal environment. Science of the Total Environment, 882-891. doi: https://doi.org/10.1016/j.scitotenv.2017.11.327

Yang, C., He, X., Yu, L., Yang, J., Yan, F., Bu, K., Zhang, S. (2017). The cooling effect of urban parks and its monthly variation in a snow climate city. Remote Sensing, 9(10), 1066. doi: https://doi.org/10.3390/rs9101066

Yuan, J., Emura, K., \& Farnham, C. (2017). Is urban albedo or urban green covering more effective for urban microclimate improvement?: A simulation for Osaka. Sustainable Cities and Society (32), 78-86. doi: http://dx.doi.org/10.1016/j.scs.2017.03.021. 